WATER-QUALI'TY ASSESSMENT OF FRANCIS E. WALTER RESERVOIR, LUZERNE ANI) CARBON COUNTIES, PENNSYLVANIA

By James L. Barker

U.S. GEOLOGLCAL SURVEY

Water-Resuirces Investigation Report 33-4032

Prepared in cooperation with the

U.S. ARMY CORPS OF ENGINEERS,

PHILADELPHIA DISTRICT

June 1983 
UNITED STATES DEPARTMENT OF THE INTERIOR

JAMES G. WATT, Secretary

GEOLOGICAL SIJRVEY

Dallas L. Peck, Director

For additional information write to:

District Mief

U.S. Feological Survey

4th Floor, Federal Bullding

P. O. Box 1107

Harrisburg, Pennsylvania $17108-1107$
Coples of this report can be purchased from:

Open-File Service Section Western Distribution Branch U. S. Geological Survey Box 25425, Federal Center Lakewood, colorado 80225 (Telephone: [303] 234-5888) 


\section{ILLUSTRATIONS}

Figure 1.--Map showing location of Francis E. Walter Reservoir......... 2

2.--Map showing Francis E. Walter Reservoir showing existing and proposed water levels, major tributaries and waterquality sampling sites............................... 5

3.--Graphs showing flow-duration curves of daily discharges...... 11

4.--Graph showing comparison of average monthly streamflow with streamflow on days of sampling, Lehigh River at Stoddartsville................................... 13

5.--Graph showing precipitation departure from normal........... 13

6.--Graph showing nitrogen and phorphorus transport curves for tributary streams................................... 22

7.--Graphs of sediment-transport curves for tributary streams.... 28

8.--Graph showing plots of equal temperature, spillway pool

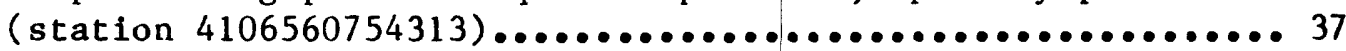

9.--Graph showing plots of equal dissolved oxygen, spillway pool (station 4106560754313).............................. 37

10.--Graphs showing dissolved oxygen and temperature profiles in the Bear Creek tributary arm of reservoir, station 5........ 38

11.--Graphs showing dissolved oxygen and temperature profiles in Lehigh River tributary arm of reservoir, station $6 \ldots \ldots \ldots \ldots \ldots . .38$

12.--Graph showing influence of phytoplankton density on Secchi disc visibility................................. 40

13.--Graph showing depth-time diagram of plots of equal $\mathrm{pH}$ at spillway pool site in 1981.............................. 40

14.--Graph showing total phosphorus loading versus the ratio of mean depth $(\ddot{z})$ to hydraulic residence time (TW) and its relation to the eutrophication potential of a lake...........

TABLES

Table 1.--Summary of historical water-quality data collected by U. S.

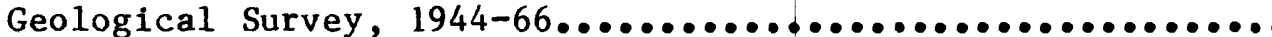

2.--Summary of historical water-quality data collected by Pennsylvania Department of Environmental Resources, 1976-78.......................................

3.--Summary of historical water-quality data collected by consultants, 1975-80................................

4.--List of water-quality sampling sites in Francis E. Walter Reservoir drainage basin................................ 9

5.--Water balance for Francis E. Walter Reservoir.............. 12

6.--Stream water-quality data collected from June 1981 to

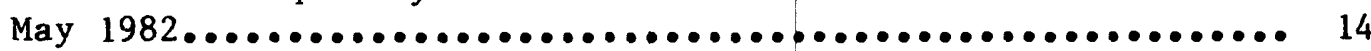

7.--Statistical summary of stream water-quality data collected from June 1981 to May $1982 \ldots \ldots \ldots \ldots \ldots \ldots \ldots \ldots \ldots \ldots \ldots \ldots \ldots \ldots \ldots \ldots \ldots$

8.--Long-term annual averages of total nitrogen transported by tributaries to Francis $E_{\text {. Walter Reservoir and from }}$ reservoir to to Lehigh River.............................. 
Table 9.--Long-term averages of total phosphorus transported by

Page tributaries to Francis E. Walter Reservoir and from reservoir to Lehigh River................................

10.--Summary of dissolved trace metals analyses at station 014478.00 - Lehigh River near White Haven,

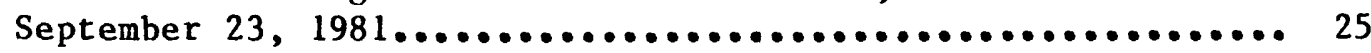

11.--Summary of fecal coliform and fecal streptococcus analysis... 26

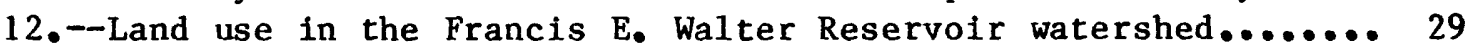

13.--Reservoir water-quality data collected from June 1981 to May 1982........................................ 30

14.--Statistical summary of reservoir water-quality data, June 1981 to May $1982 \ldots \ldots \ldots \ldots \ldots \ldots \ldots \ldots \ldots \ldots \ldots \ldots \ldots \ldots \ldots \ldots \ldots \ldots . \ldots \ldots$

15.--Summary of phytoplankton composition at spillway pool,

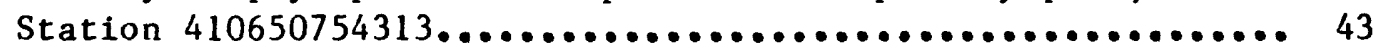

16.--Summary of phytoplankton composition at Bear Creek pool,

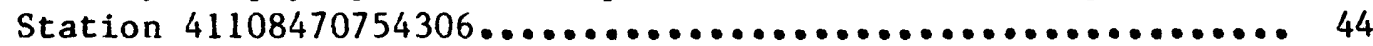

17.--Summary of phytoplankton composition at Lehigh River pool, Station $410750753947 \ldots \ldots \ldots \ldots \ldots \ldots \ldots \ldots \ldots \ldots \ldots \ldots \ldots \ldots \ldots \ldots \ldots \ldots$

FACTORS FOR CONVERTING U.S. CUSTOMARY UNITS TO INTERNATIONAL SYSTEM OF UNITS (SI)

Mulitiply inch-pound units

inches (in)

feet ( $f t$ )

miles (mi)

acres

square miles $\left(\mathrm{mi}^{2}\right)$

acre-feet (acre-ft)

cubic feet per second $\left(\mathrm{ft}^{3} / \mathrm{s}\right)$

pounds per acre (1bs/acre)

tons (short)
By

$$
\begin{gathered}
25.4 \\
0.3048 \\
1.609 \\
4.047 \times 10^{-3} \\
2.590 \\
1.233 \times 10^{-6} \\
0.02832 \\
1.1208 \\
0.9072
\end{gathered}
$$

To obtain SI units

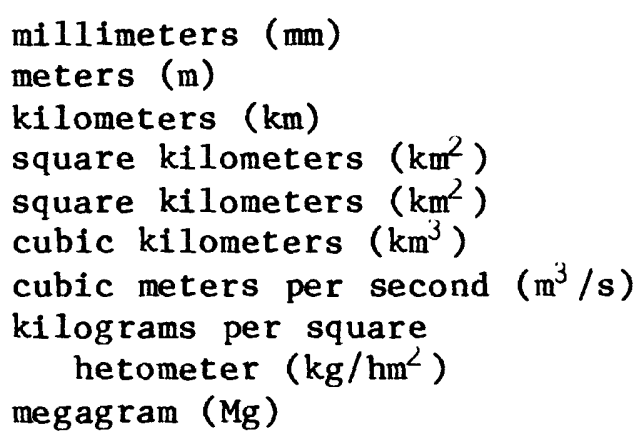

DATUM

National Geodetic Vertical Datum of 1929 (NGVD of 1929): a geodetic datum derived from a general adjustment of the first-order level nets of both the United States and Canada, formerly called mean sea level. NGVD of 1929 is referred to as sea level in this report. 


\title{
WATER-QUALITY ASSESSMENT OF FRANCIS E. WALTER RESERVOIR LUZERNE AND CARBON COUNTIES, PENNSYLVANIA
}

\author{
James L. Barker
}

\section{ABSTRACT}

Water-quality data, both past and present, show that the waters of the upper Lehigh River basin are somewhat acidic, but otherwise are generally of good quality. This report contains a summary of all known water-quality data* collected by the U. S. Geological Survey and other agencies, as well as a synopsis of current water-quality conditions in the reservior and its tributaries.

Water-quality data collected from June 1981 to May 1982 indicate that raising the pool level from 1,300 to approximately 1,392 feet above sea level (NGVD of 1929) has had some significant, if only temporary, detrimental impacts on the reservoir system and its discharge. Depth profile measurements show that, while the impoundment was thermally stratified for only about 2 weeks, the dissolved oxygen concentrations were depressed to levels critical to fishlife throughout much of the reservoir. Another effect of the raised pool was the lowering of $\mathrm{pH}$ in the impounded water. Median $\mathrm{pH}$ values were less than 6.0 throughout the reservoir, whereas they commonly exceeded 6.5 at the normal pool elevation. Tests for fecal coliform and fecal streptococcus indicate the impounded water is nearly free of enteric bacteria. Algal analyses and nutrient concentrations support the premise that the impoundment is nutrient poor and phosphorus limited.

Raising the water level an additional 125 feet should have no permanent detrimental effect upon water quality and will greatly increase available habitat for fish and waterfowl. Increased retention time should not alter the current trophic status and may decrease the concentration of available nutrients.

\section{INTRODUCTION}

\section{Study Area}

Francis E. Walter Reservoir, originally known as Bear Creek Reservoir, is on the boundary of Luzerne and Carbon Counties (Fig. 1). It is a manmade impoundment created in 1961 by damming the Lehigh River at the confluence with Bear Creek. The $289 \mathrm{mi}^{2}$ drainage basin above the dam is predominantly forested with little development; however, recent commercial enterprises such as a ski area and a growing shopping plaza, and increasing numbers of permanant and seasonal homes have increased the potential for environmental degradation.

The reservoir is currently operated as a flood control project with incidental recreation benefits that include fishing and white-water boating. 


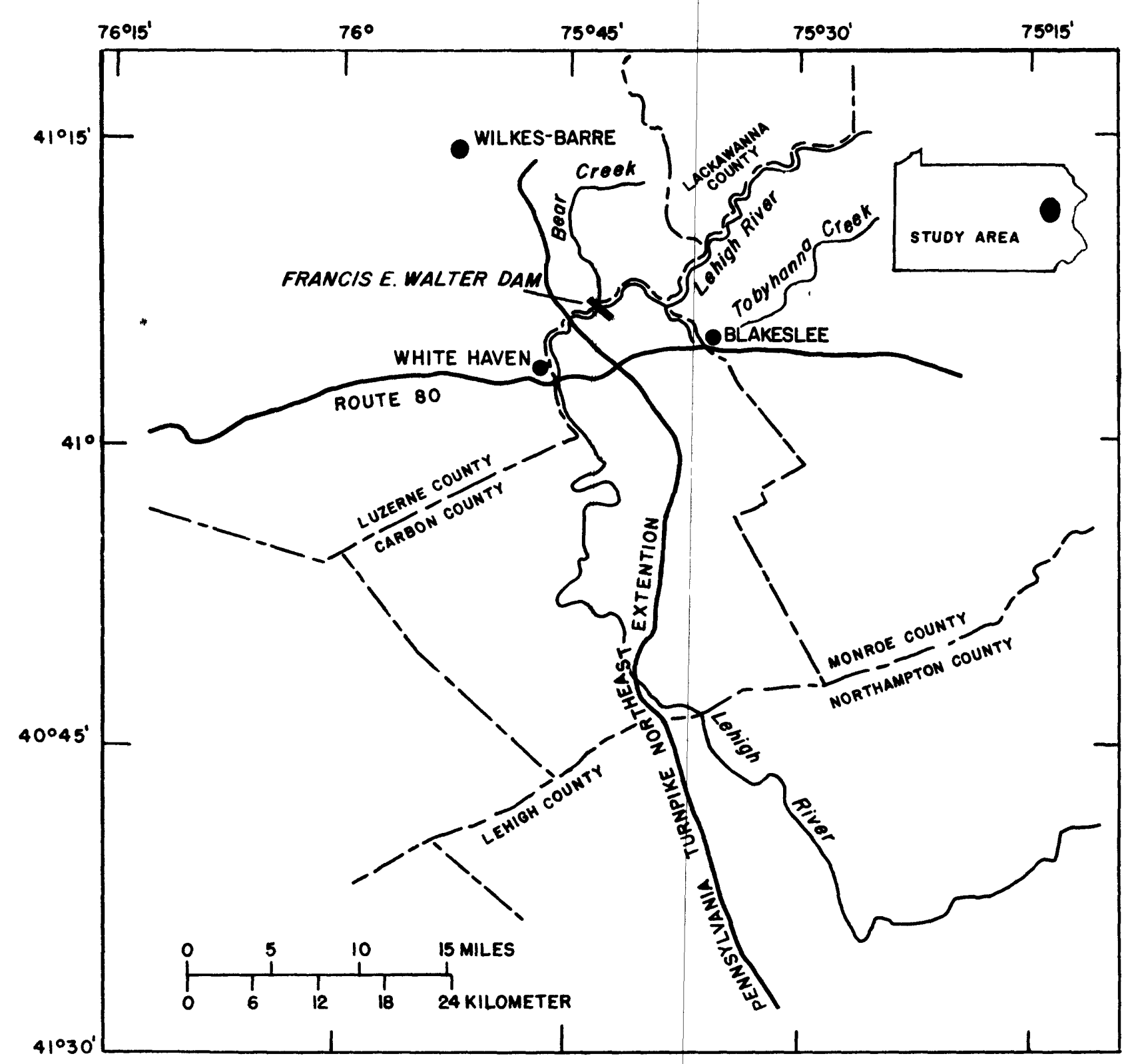

Figure 1.--Location of Francis E. Walter reservoir. 
The principal objectives of this study were to assess the past and current water quality of the impoundment, its major tributaries, and its discharge, and t) address the environmental impact of raised pool levels. Data collection was directed toward documenting the temporal and spatial variations in physical, chemical, and biological characteristics of the water, to estimate annual nutrient and suspended sediment loading, and to determine the algal biomass, dorinant genera, algal growth potential, and limiting nutrient.

\section{Previous Studies}

Water chemistry and biological data for streams in the upper Lehigh River basin dating back to 1944 provide a historical record and basis for comparing present dati. The earlter published data that were intermittantly collected from 1944 to 1966 are found in U.S. Geological Survey Water-Supply Paper 1879-H by McCarren and Keighton (1969). These analyses consisted of what were then known as a "standard complete analysis," and consisted of the najor cations and anions, dissolved solids, hardness, total acidity, speciftc conductance, $\mathrm{pH}$, and color. The results of these analyses, presented in table 1 , indicate the water entering the reservolr from Bear and Tobyhanna Creeks and the Lehigh River was of excellent quality - $\mathrm{pH} 5.3$ to 7.1 and specific conductance ranged from 22 to 57 microrahos.

In 1965 and 1966 field studies of the Lehigh River drainage basin were conducted in a cooperative study by the Delaware River Basin Commission, the Pennsylvania Fish Comission, and the Pennsylvania Department of Health. "The prinary objective of this investigation was to determine the relation between water quality of the Lehigh River and its biological productivity." (Pollison and Craighead, 1968). The investigation included the quantitative and qualttative measurement of macroinvertebrates, fishes, and vascular plants. Physical and chemical properties were also determined. The report concludes that from the headwaters to White Haven "the chemical and biological results indicate a high level of water quality and low productivity of the biota."

The Penusylvania Departinent of Environuental Resources (PaDER) conducted a water-quality sampling progran fror January 1976 to Novenber 1978 at station 01447500 (Lehigh Rijer gage at Stoddartsville) and Station 014477.20 (Tobyhanna Creek gage near Blakeslee, fig. 2). These analyses were done three to five times a year. The PaDER analyses indicate there has been little, if any, change in water quality over the past 40 years. The maintenance of high quality water has been largely due to the slow development of the basin. The results are summarized in table 2.

Beginning in 1975 and continuing through 1980, varlous monitoring programs by private laboratories under contract to the U.S. Army Corps of Engineers, Philadelphia District, have collected and analyzed water samples from the upper Lehigh River drainage basin. These samples were collected monthly during the nongrowing season and biweekly during the growing season. The results of this long-term data-collection prograin are presented in the form of a statistical summary in table 3. The raw data are on file at the corps of Engineers, Philadelphia District. 


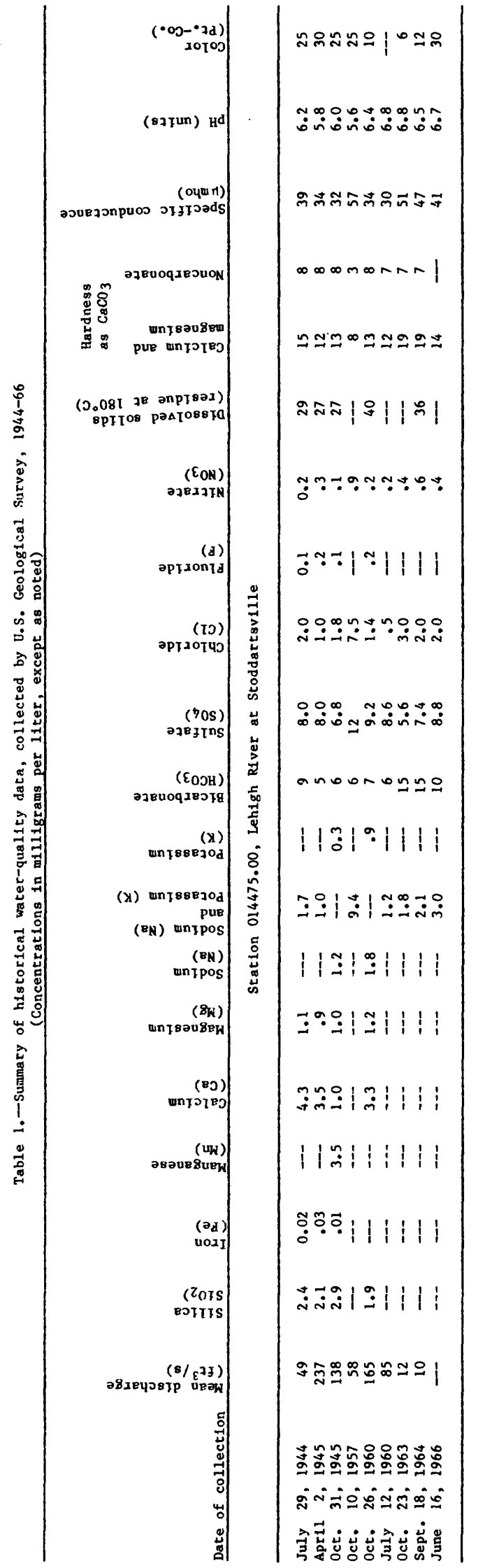

\begin{tabular}{|c|c|c|c|c|}
\hline 드임 $\left.\right|^{\infty}$ 요요 & & $\left.\cong\right|^{\operatorname{and} \pi n}$ & & 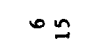 \\
\hline 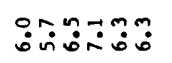 & & 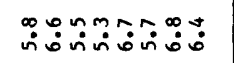 & & ( \\
\hline 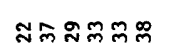 & & 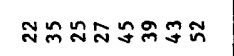 & & $\approx m$ \\
\hline noomm | & & nunanantat & & on \\
\hline$\subseteq^{\infty} 9=\approx 0$ & & 오용ㅇㅇㅛ & & $\cong \cong$ \\
\hline$\approx|1| 2 \mid$ & & $\approx \approx n \| 111=$ & & $\mid \vec{m}$ \\
\hline 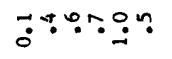 & & • & a & 号 \\
\hline :11111 & & $\ddot{0} 1111111$ & ฐँ๊ & 11 \\
\hline 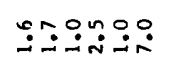 & 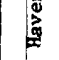 & 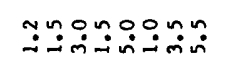 & 剂 & 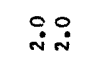 \\
\hline 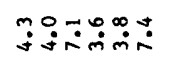 & gू & 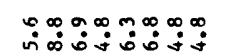 & $\Phi$ & 冓 \\
\hline ogngon & שัّ & $N N+N \infty+\infty N$ & 这 & $\infty \infty$ \\
\hline 11111 & 冓 & 1111111 & $\frac{3}{9}$ & 11 \\
\hline m̃ & ت & 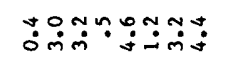 & 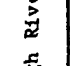 & 遇 \\
\hline 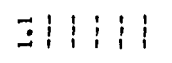 & 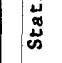 & 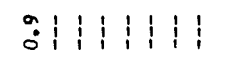 & $\$$ & $i$ \\
\hline $\begin{array}{l:llll}\infty & 0 & 1 & 1 & 1 \\
0 & 1 & 1 & 1 & 1\end{array}$ & & 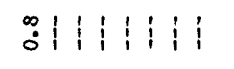 & $\frac{0}{9}$ & 1 \\
\hline 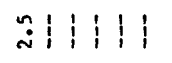 & & 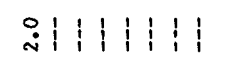 & 通 & 11 \\
\hline 111911 & & $11: 11111$ & & $1 !$ \\
\hline 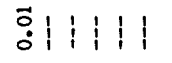 & & 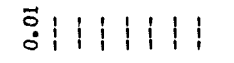 & & 1 \\
\hline$\stackrel{\infty}{9} 111111$ & & îl|11111 & & 11 \\
\hline 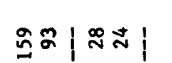 & & 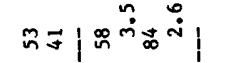 & & $\overrightarrow{0} \overrightarrow{5}$ \\
\hline 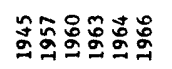 & & 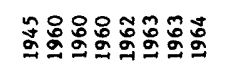 & & 惫す \\
\hline & & ตี่ง & & $\sin ^{\circ}$ \\
\hline 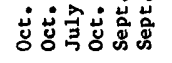 & & 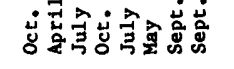 & & 岁离 \\
\hline
\end{tabular}




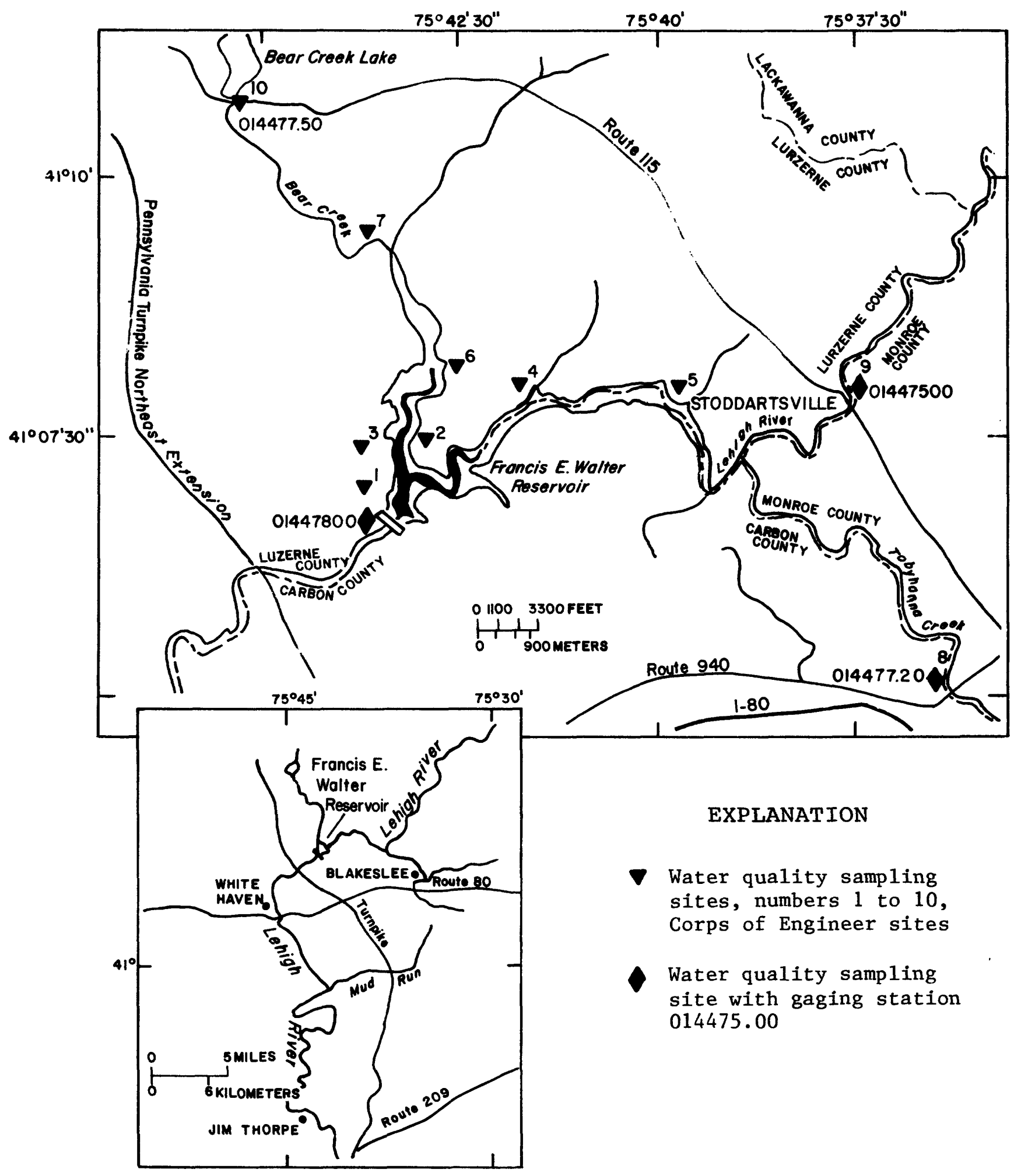

Figure 2.--Francis E. Walter Reservoir showing exhisting and proposed water levels, major tributaries, and water quality sampling sites. 


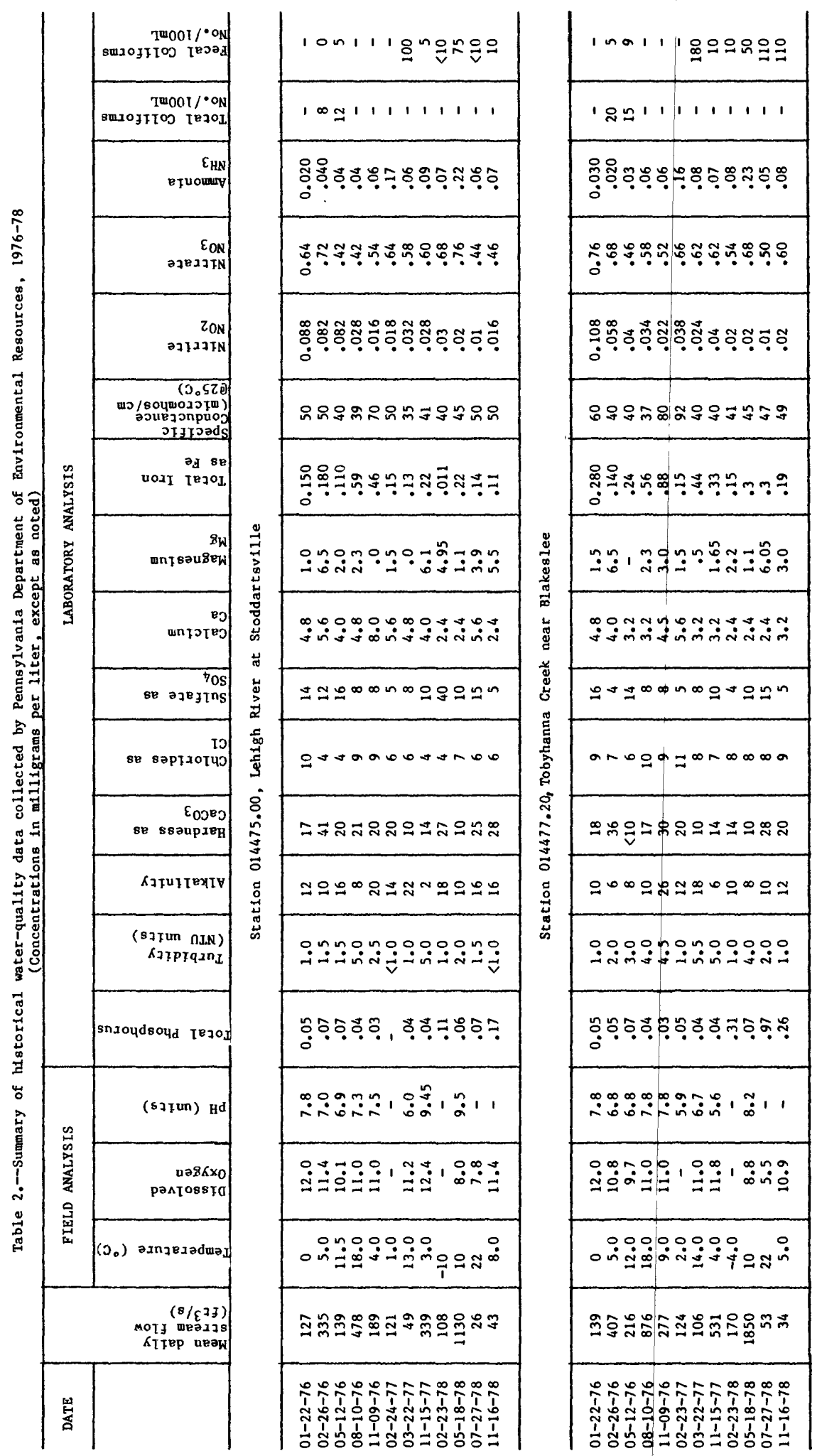


Table 3.--Summary of historical water-quality data collected by consultants, $1975-80^{1}$

(Concentrations in milligrams per 1iter, except as noted)

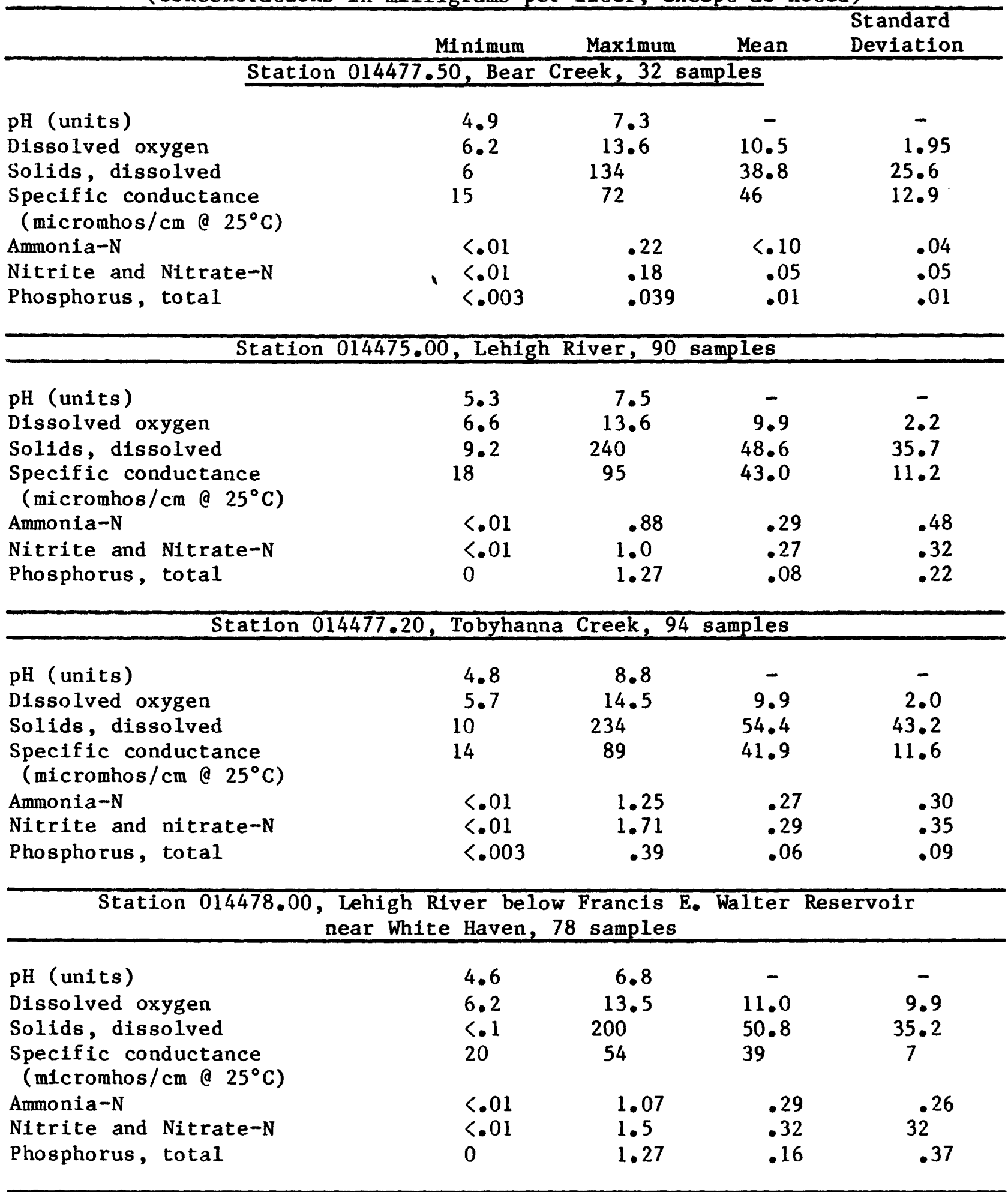

Buchart-Horn, York, PA;

Betz-Converse-Murdoch, Inc, Plymouth Meeting, PA. 
In July 1975, the Pennsylvania Fish Commission conducted a survey at Francis E. Walter Reservoir that consisted of profiling the deepest part of the reservoir for temperature, dissolved oxygen, $\mathrm{pH}$, and alkalinity. These data, which are also on file at the Corps of Engineers office, indicate two significant facts: the reservoir did not thermally stratify and the dissolved oxygen content remained high throughout the water column.

In June 1978, the Geological Survey completed a 1-year Investigation to determine the loading and average annual transport of phosphorus to the Francis E. Walter Reservoir from gaged and ungaged tributaries. The results indicated an areal loading rate of $15.3 \mathrm{~g} \mathrm{P} / \mathrm{m}^{2} / \mathrm{yr}$, and an average annual transport of approximately 6 tons. These estimates may be low because of insufficient highflow data. Studies by Vollenwelder (1975), indicate reservoirs similiar to Francis $\mathrm{E}$. Walter Reservoir remain relatively low in nutrients because of the short retention time.

\section{Materials and Methods}

Water quality of the tributaries was measured during scheduled monthly field trips and unscheduled storm sampling beginning June 23, 1981. Table 4 lists the Geological Survey and Corps of Engineers sampling stations. The sampling stations on the Lehigh River and Tobyhanna Creek tributaries coincide with stream-gaging stations maintained and operated by the U.S. Geological Survey.

Stream measurement of temperature, dissolved oxygen, $\mathrm{pH}$, and specific conductance were made at all stream sampling stations with a Hydrolab Series $4041^{1}$ multiparameter monitor.

Water samples for chemical analyses were collected with a Kemmerer type water sampler and placed in clean plastic bottles. Water samples for dissolved constitutents were filtered through 0.45 micron filters. Nitrogen and phosphorus samples were preserved with mercuric chloride and chilled to $4^{\circ} \mathrm{C}$ until analyzed at the U.S. Geological Survey Atlanta Central Laboratory. Inorganic chemical constitutents were determined by methods described by Skougstad and others, (1979), biological constituents by methods described by Greeson and others, (1977), and suspended sediment by methods described by Guy (1969).

Suspended sediment, total phosphorus, and total nitrogen loading from the three major tributaries were calculated by the flow-duration-curve method described by Miller, (1951). This method of computation provides long-term average transport and is the method the U.S. Geological Survey has been using for years to determine the suspended-sediment transport in streams based on a few observations. The method is widely accepted as the most accurate, short of datly sampling.

1 The use of brand names in this report is for identification purposes only and does not constitute endorsement by the U.S. Geological Survey. 
Table 4.--Water-quality sampling sites in Francis E. Walter Reservoir drainage basin

\begin{tabular}{|c|c|c|}
\hline $\begin{array}{l}\text { U.S. Geological } \\
\text { Survey station } \\
\text { number }\end{array}$ & $\begin{array}{c}\text { U.S. Army Corps of } \\
\text { reference } \\
\text { number }\end{array}$ & Location \\
\hline 014477.50 & 7 & Bear Creek near White Haven, Pa. \\
\hline 014477.20 & 8 & $\begin{array}{l}\text { Tobyhanna Creek near Blakeslee, } \\
\text { Pa. }\end{array}$ \\
\hline 014475.00 & 9 & $\begin{array}{l}\text { Lehigh River at Stoddartsville, } \\
\text { Pa. }\end{array}$ \\
\hline 014478.00 & outf low & $\begin{array}{l}\text { Lehigh River below Francis E. } \\
\text { Walter Reservolr near White } \\
\text { Haven, } \mathrm{Pa} \text {. }\end{array}$ \\
\hline 410656075431300 & 1 & Spillway pool near surface. \\
\hline 410656075431301 & & Spil1way pool near bottom. \\
\hline 410847075430600 & 6 & Bear Creek arm near surface. \\
\hline 410847075430601 & & Bear Creek arm near bottom. \\
\hline 410745075394700 & 5 & Lehigh River arm near surface. \\
\hline 410754075394701 & & Lehigh River arm near bottom. \\
\hline
\end{tabular}


Samples for bacterial analyses were collected several inches below the surface at midstream from the three major inflow tributaries. They were collected in $250-\mathrm{mL}$ sterile polypropalene bottles and Immediately chilled to $4^{\circ} \mathrm{C}$. The analyses were done by field personnel within 2 hours using membrane filtration techniques in accordance with methods described by Greeson and others, (1977).

Water quality at the three reservoir stations was characterized by biweekly depth profiles from April 9, 1981 to November 4, 1981. Stream measurenents of temperature, dissolved oxygen, $\mathrm{pH}$, and specific conductance, were determined at the lake sites with a multiparameter Hydrolab Corporation Serfes 7000 waterquality monitor by the U.S. Army Corps of Engineers. Measurements were obtained at 5-foot intervals. The reservoir stations are shown in figure 2. In addition to collecting near surface samples at all three reservoir stations for nutrient, bacterlological, and algal analyses, additional water samples were collected near the botton for nutrient analyses. Transparencies were obtained with a standard 20-cm-diameter Secchi disk attached to a calibrated metal 1ine.

\section{WATER QUALITY OF STREAMS}

\section{Hydrology}

Stream-sampling stations were selected on the basis of their proximity to existing stream-gaging stations and the reservoir. Stream-gaging stations are raintained by the U.S. Geological Survey on Tobyhanna Creek near Blakeslee (station 014477.20), the Lehigh River at Stoddartsville (station 014475.00), and on the Lehigh River near White Haven (station 014478.00), downstream of the dam. The fourth strean-sampling site (station 01477.50) is on Bear Creek near White Haven. Flow-duration curves developed by the U.S. Geological Survey for the Lehigh River, Tobyhanna Creek, and Bear Creek are shown in figure 3.

The drainage area at the outlet of Frances E. Walter Reservoir is $289 \mathrm{mi}^{2}$. The size of the drainage of each major tributary and its contribution to the annual flow is summarized in table 5. Note that 87 percent of the annual inflow is gaged.

As shown in figure 4, the beginning of the 10-month sampling period was relatively dry. Streamflow on the dates sampled in July through October was less than the 4-year monthly average. The sample in late October represented a storm sample.

Additional evidence of lower-than-normal streamflows prior to and during the sampling period is presented in figure 5 from precipitation data gathered at the National Weather Service station at Francis E. Walter Reservoir. The departure below normal precipitation during 1980 was about 16 inches and during 1981, about 7 inches.

The remainder of this section is concerned with selected measurements related to the current water quality of tributary and outflow water. A listing of the chemical, physical and biological data collected at these sites is given in table 6 . A statistical summary of these data is presented in table 7 . 


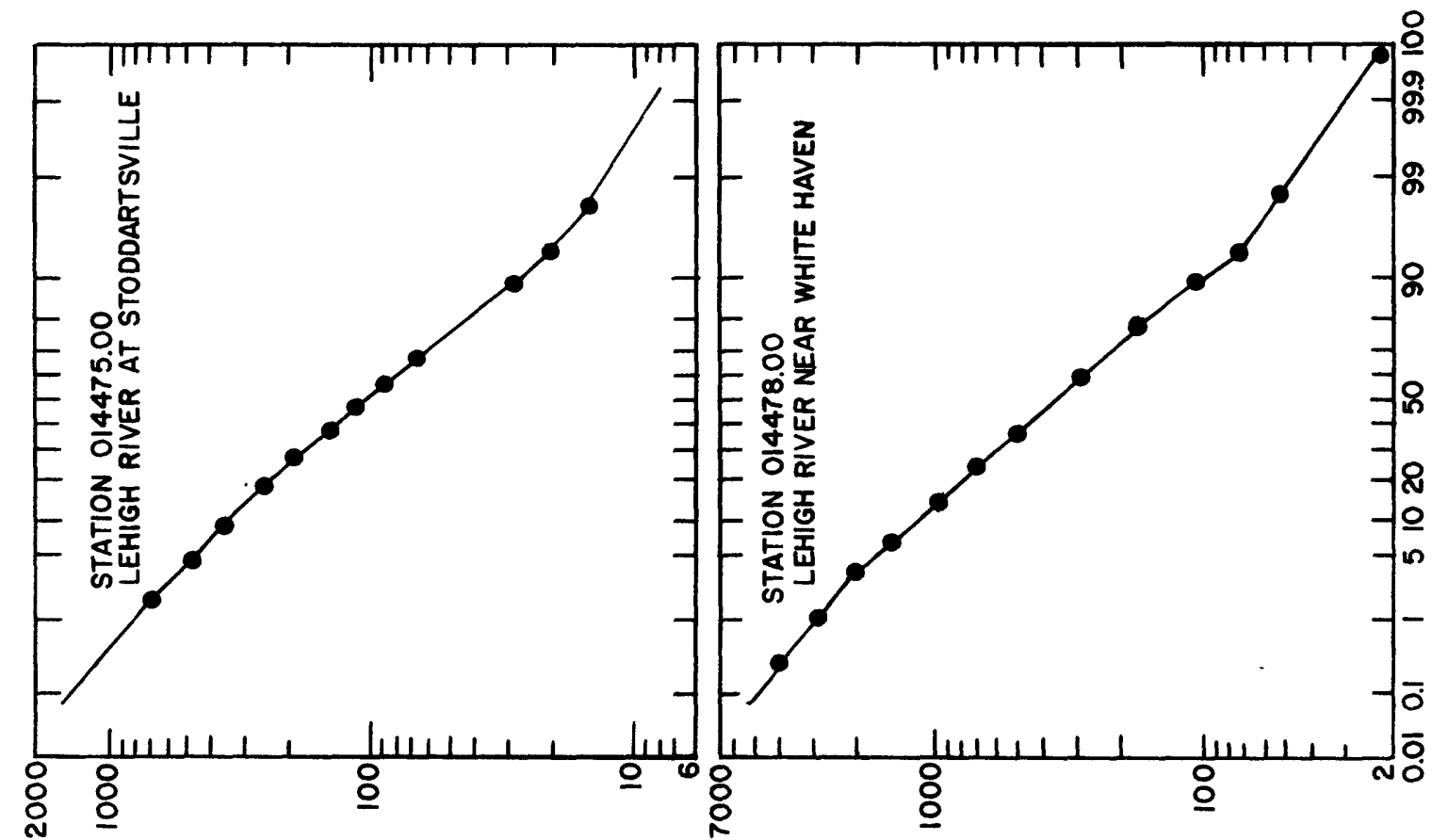

亗

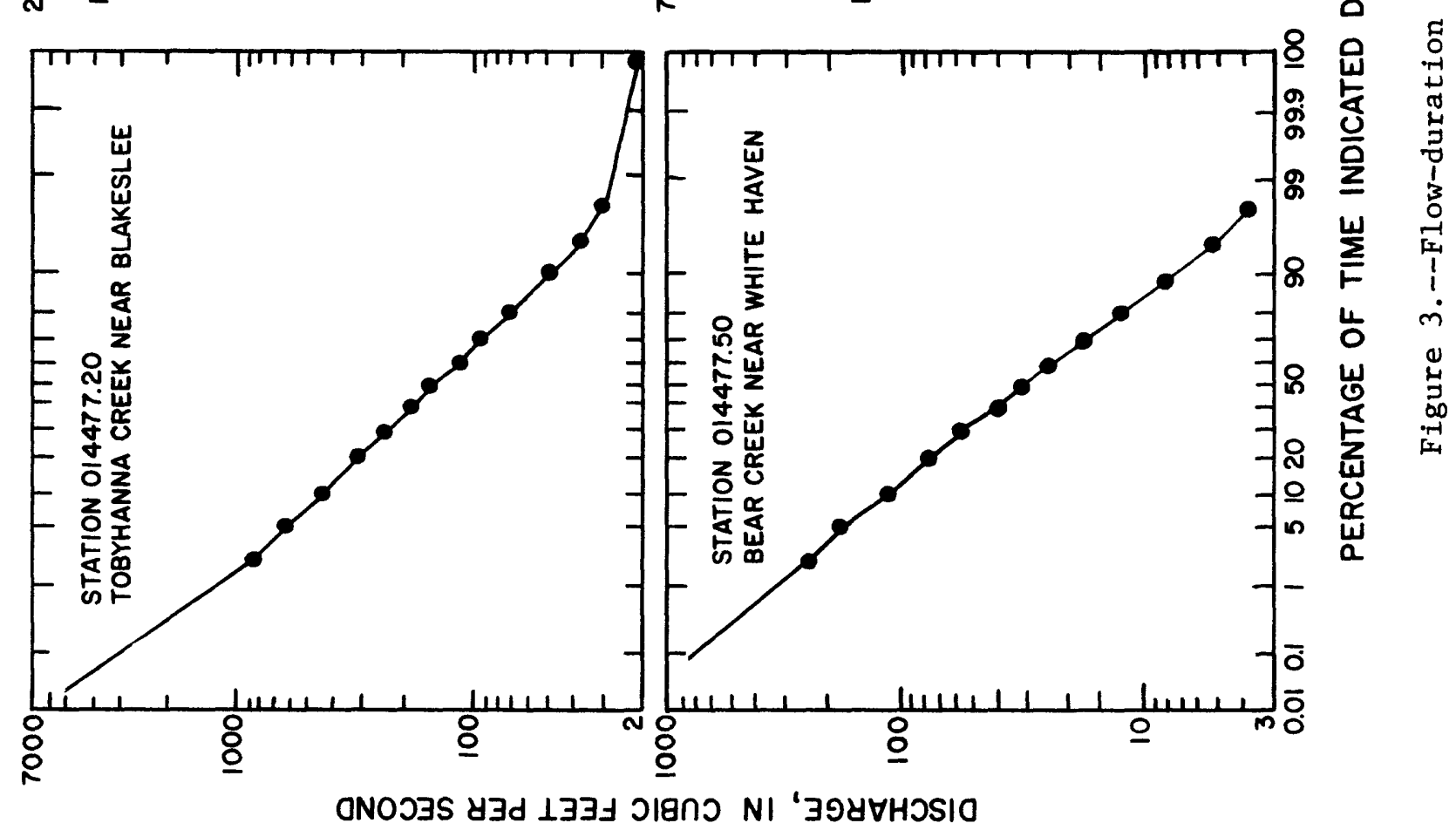




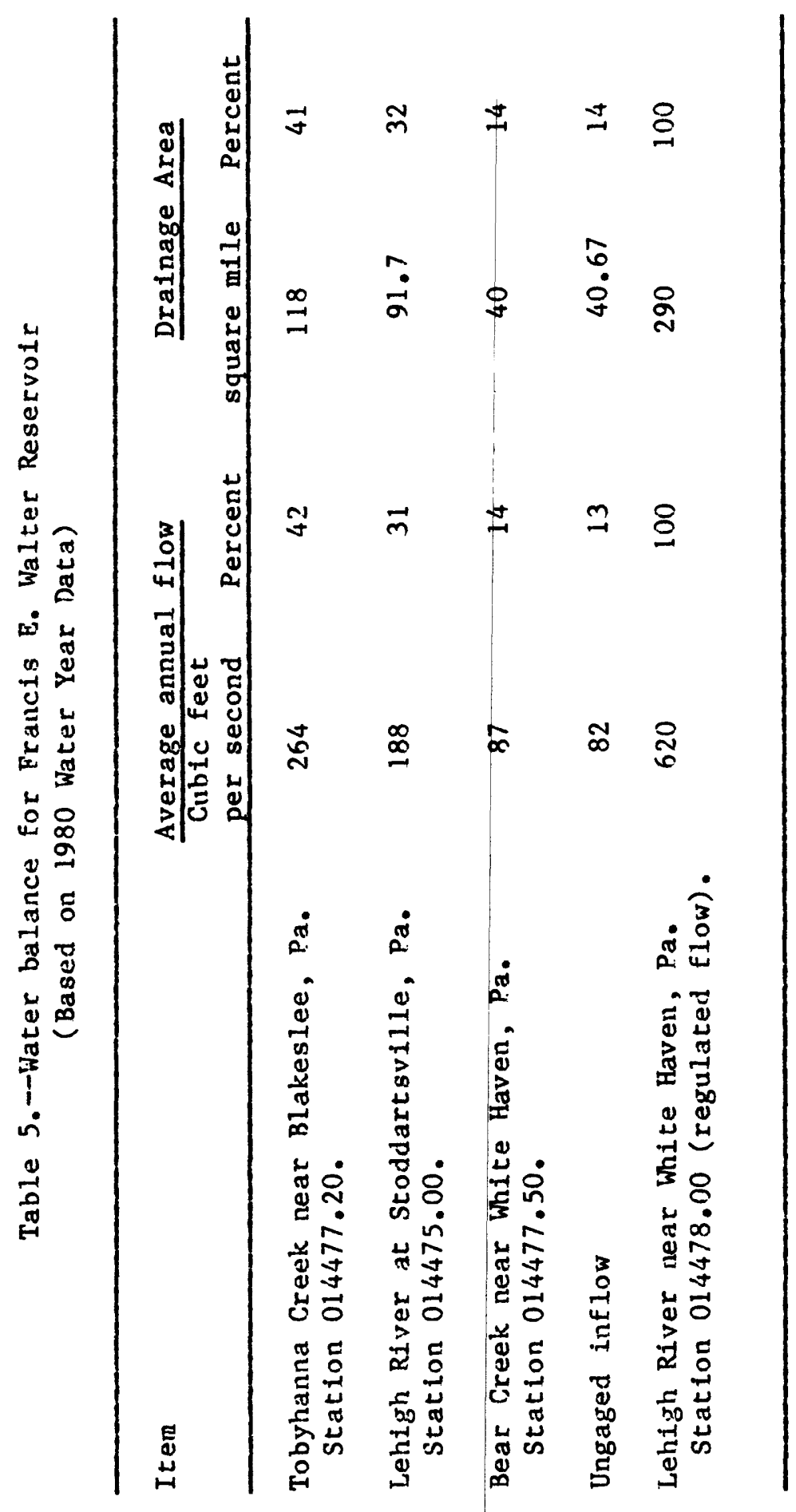




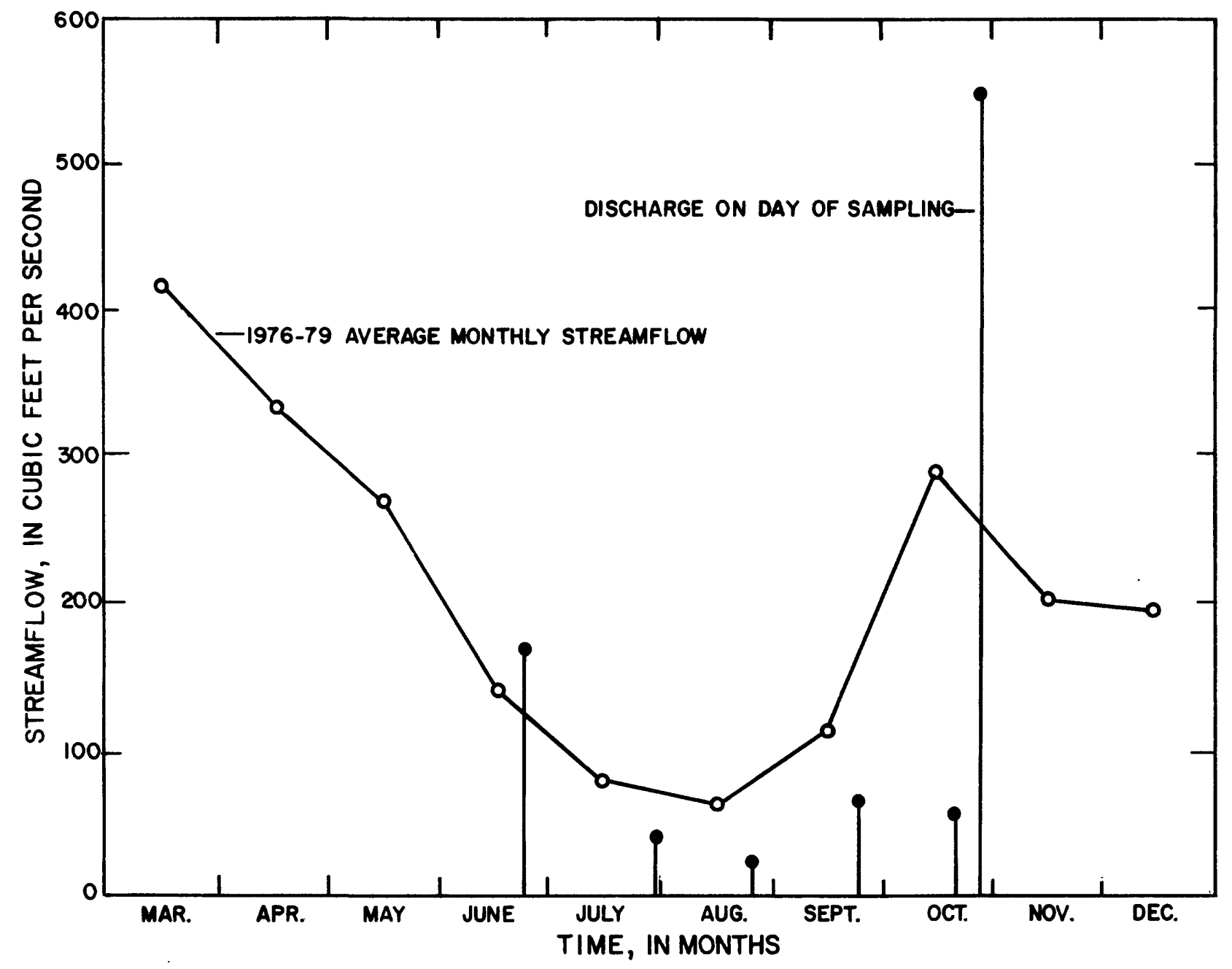

Figure 4.--Comparison of average monthly streamflow with streamflow on days of sampling, Lehigh River at Stoddartsville.

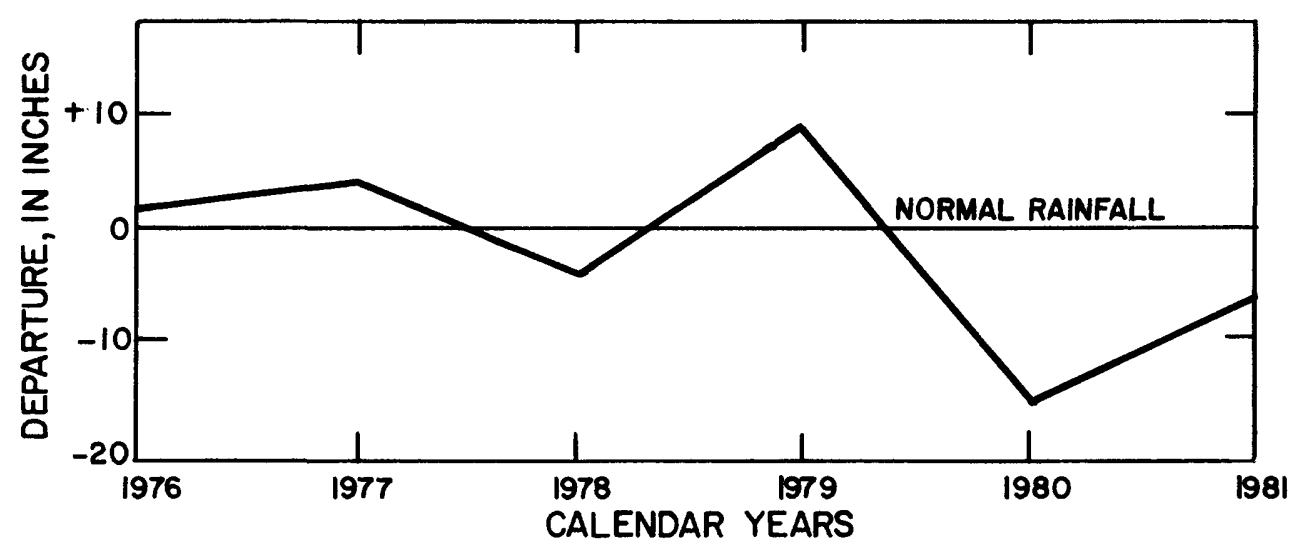

Figure 5.--Precipitation departure from normal. Data from National Weather Service Station at Francis E. Walter Reservoir. 


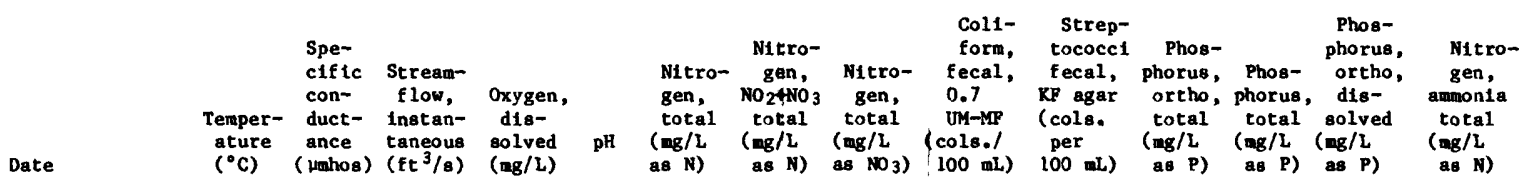

Station 014475.00, Leh1gh River at Stoddartioville, Pa.

1981:

\begin{tabular}{|c|c|c|c|c|c|c|c|c|c|c|c|c|c|c|c|}
\hline $\begin{array}{l}\text { June } \\
\text { July }\end{array}$ & $\begin{array}{l}23 \ldots \\
28 \ldots\end{array}$ & $\begin{array}{l}18.5 \\
19.0\end{array}$ & $\begin{array}{l}39 \\
41\end{array}$ & $\begin{array}{r}172 \\
49\end{array}$ & $\begin{array}{l}9.4 \\
9.2\end{array}$ & $\begin{array}{l}6.4 \\
6.0\end{array}$ & $\begin{array}{r}0.71 \\
.28\end{array}$ & 0.11 & $\begin{array}{l}3.1 \\
1.2\end{array}$ & $\begin{array}{l}75 \\
29\end{array}$ & $\begin{array}{r}{ }^{1} \times 500 \\
250\end{array}$ & $\begin{array}{r}0.017 \\
.008\end{array}$ & $\begin{array}{r}0.039 \\
-\end{array}$ & $\begin{array}{r}0.005 \\
.000\end{array}$ & $\begin{array}{r}<0.010 \\
.010\end{array}$ \\
\hline Aug. & $25 \ldots$ & 20.9 & 46 & 25 & 10.0 & 7.9 & .71 & .05 & 3.1 & 10 & 80 & .022 & .023 & .006 & .240 \\
\hline Sept. & $23 . \ldots$ & 12.1 & 46 & 73 & 10.3 & 5.9 & .51 & .10 & 2.3 & 55 & 50 & .012 & .017 & .005 & .03 \\
\hline oct. & $20 \ldots$ & 6.1 & 36 & 63 & 11.0 & 5.8 & .41 & .05 & 1.8 & 9 & 1 & .005 & .003 & .005 & $<.01$ \\
\hline Jan. & $28 \ldots$ & 0.1 & 32 & 203 & 13.8 & 5.8 & .64 & .25 & 2.8 & - & - & .005 & .013 & .005 & .05 \\
\hline Apr Il & $05 \ldots$ & 2.8 & 29 & 604 & 14.1 & 5.6 & .54 & .11 & 2.4 & - & -- & .008 & .009 & .004 & .06 \\
\hline May & 04. & 12.8 & 35 & 180 & 10.4 & 5.7 & .51 & .07 & 2.2 & $<1$ & K18 & $<.010$ & .016 & $<.010$ & \\
\hline
\end{tabular}

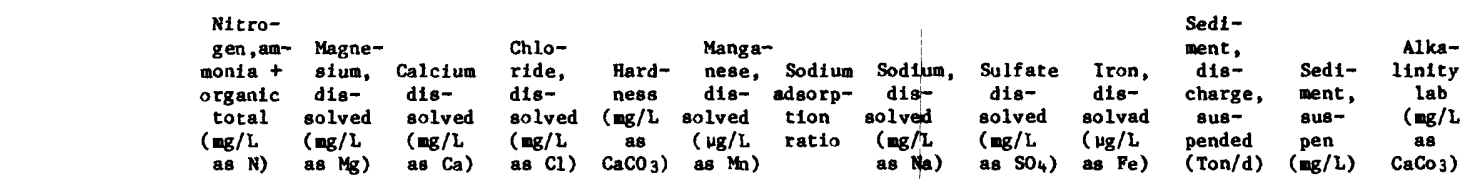

1981:

\begin{tabular}{|c|c|c|c|c|c|c|c|c|c|c|c|c|c|c|}
\hline Juna & $23 . \ldots$ & 0.60 & - & - & - & -- & - & - & - & -- & - & 3.3 & 7 & - \\
\hline July & $28 .$. & .27 & -- & -- & - & -- & - & - & - & -- & - & - & -- & - \\
\hline Sept. & $23 . .$. & .41 & 1.1 & 4.7 & 4.4 & 16 & 20 & 0.3 & 2.4 & 7.6 & 90 & .20 & 1 & - \\
\hline Oct. & $20 . .$. & .36 & - & - & -- & $\cdots$ & $\cdots$ & -- & - & - & - & .17 & 1 & - \\
\hline Jan. & $28 \ldots$ & .39 & -- & $\cdots$ & - & - & - & - & -- & - & -- & - & -- & - \\
\hline April & $05 \ldots$ & .43 & - & $\cdots$ & 3.7 & - & - & - & -- & 8.4 & - & - & -- & 3.0 \\
\hline May & $04 \ldots$ & .44 & -- & $-\infty$ & - & - & - & -- & -- & -- & -- & -- & -- & - \\
\hline
\end{tabular}

May $04 . .$.

Date

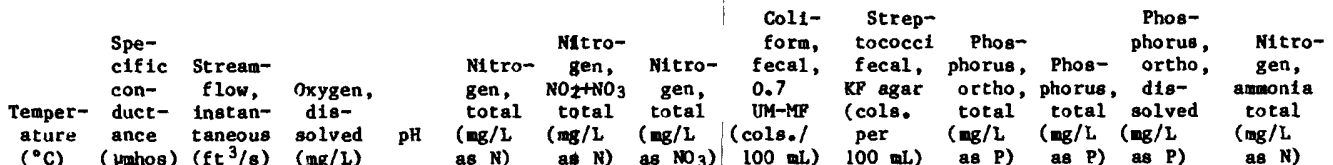

Station 014477.20 , Tobyhanna Creek near Blakealee, Pa.

1981:

\begin{tabular}{|c|c|c|c|c|c|c|c|c|c|c|c|c|c|c|c|}
\hline $\begin{array}{l}\text { June } \\
\text { July } \\
\text { Aug. } \\
\text { Sept. } \\
\text { Oct. } \\
\text { Oct. } \\
\text { B2: }\end{array}$ & $\begin{array}{l}23 \ldots \\
28 \ldots \\
25 \ldots \\
23 \ldots \\
20 \ldots \\
28 \ldots\end{array}$ & $\begin{array}{r}18.5 \\
18.8 \\
17.4 \\
12.9 \\
5.5 \\
--\end{array}$ & $\begin{array}{l}39 \\
33 \\
36 \\
37 \\
33 \\
49\end{array}$ & $\begin{array}{r}306 \\
191 \\
269 \\
120 \\
78 \\
459\end{array}$ & $\begin{array}{r}8.6 \\
8.6 \\
8.9 \\
10.1 \\
11.0 \\
\end{array}$ & $\begin{array}{l}5.8 \\
5.7 \\
5.9 \\
5.6 \\
5.8 \\
5.9\end{array}$ & $\begin{array}{r}0.54 \\
.53 \\
.41 \\
.67 \\
.82 \\
.31\end{array}$ & $\begin{array}{l}0.06 \\
.07 \\
.08 \\
.09 \\
.05 \\
.08\end{array}$ & $\begin{array}{l}2.4 \\
2.3 \\
1.8 \\
2.9 \\
3.6 \\
1.4\end{array}$ & $\begin{array}{r}70 \\
20 \\
18 \\
9 \\
4 \\
-\end{array}$ & $\begin{array}{r}110 \\
420 \\
154 \\
242 \\
0 \\
-\end{array}$ & $\begin{array}{r}<0.017 \\
.002 \\
.004 \\
.011 \\
.005 \\
.008\end{array}$ & $\begin{array}{r}0.024 \\
.006 \\
<.001 \\
.022 \\
.007 \\
.026\end{array}$ & $\begin{array}{r}<0.001 \\
<.001 \\
.004 \\
.005 \\
.007 \\
<.001\end{array}$ & $\begin{array}{r}<0.010 \\
<.010 \\
.020 \\
.040 \\
.050 \\
.040\end{array}$ \\
\hline $\begin{array}{l}\text { Jan, } \\
\text { April }\end{array}$ & $\begin{array}{l}28 \ldots \\
05 . \ldots\end{array}$ & 3.1 & $\begin{array}{l}34 \\
31\end{array}$ & $\begin{array}{r}224 \\
1170\end{array}$ & $\begin{array}{l}14.8 \\
13.8\end{array}$ & $\begin{array}{l}5.7 \\
4.4\end{array}$ & $\div$ & -34 & 3.1 & - & $\overline{-}$ & .005 & $\begin{array}{r}.008 \\
.018\end{array}$ & .005 & $\begin{array}{l}.060 \\
.040 \\
.020\end{array}$ \\
\hline
\end{tabular}

\begin{tabular}{|c|c|c|c|c|c|c|c|c|c|c|c|c|c|c|}
\hline $\begin{array}{l}\text { Nitro- } \\
\text { gen,an- } \\
\text { monia + } \\
\text { organic } \\
\text { total } \\
(\mathrm{mg} / \mathrm{L} \\
\mathrm{ag} / \mathrm{N})\end{array}$ & $\begin{array}{l}\text { Magne- } \\
\text { sium, } \\
\text { dis- } \\
\text { solved } \\
\text { (mg/L } \\
\text { as } \mathrm{Mg} \text { ) }\end{array}$ & $\begin{array}{l}\text { Calcium } \\
\text { dis- } \\
\text { solved } \\
\text { (ag/L } \\
\text { as Ca) }\end{array}$ & $\begin{array}{l}\text { Chlo- } \\
\text { ride, } \\
\text { dis- } \\
\text { solved } \\
\text { (ag/L } \\
\text { as Cl) }\end{array}$ & $\begin{array}{c}\text { Hard- } \\
\text { nass } \\
(\mathrm{ag} / \mathrm{L} \\
\mathrm{as} \\
\left.\mathrm{CaCO}_{3}\right)\end{array}$ & $\begin{array}{l}\text { Manga- } \\
\text { nese, } \\
\text { dis- } \\
\text { solved } \\
(\mu g / L \\
\text { as } / \mathrm{h})\end{array}$ & $\begin{array}{l}\text { Sod lum } \\
\text { adsorp- } \\
\text { tion } \\
\text { rat lo }\end{array}$ & $\begin{array}{l}\text { Sodium, } \\
\text { dijo- } \\
\text { solved } \\
\text { (mg/L } \\
\text { go } / \mathrm{Na} \text { ) }\end{array}$ & $\begin{array}{l}\text { Sulf ate } \\
\text { dis- } \\
\text { solved } \\
\text { ( } \mathrm{mg} / \mathrm{L} \\
\text { as } \mathrm{SO}_{4} \text { ) }\end{array}$ & $\begin{array}{c}\text { Iron, } \\
\text { dis- } \\
\text { solved } \\
\text { ( } \mu \mathrm{g} / \mathrm{L} \\
\text { as Fe) }\end{array}$ & $\begin{array}{l}\text { Sed1- } \\
\text { ment, } \\
\text { d1s- } \\
\text { charge, } \\
\text { sus- } \\
\text { pended } \\
\text { (Ton/d) }\end{array}$ & $\begin{array}{l}\text { Sedi- } \\
\text { ment, } \\
\text { sus- } \\
\text { pen } \\
(\text { ag } / L)\end{array}$ & $\begin{array}{c}\text { Actdity } \\
(\mathrm{mg} / \mathrm{L} \\
\mathrm{as} \mathrm{H})\end{array}$ & $\begin{array}{c}\text { Acldity } \\
\text { (ng/L } \\
\text { as } \\
\left(\mathrm{CaCO}_{3}\right)\end{array}$ & $\begin{array}{c}\text { Alka- } \\
\text { linity } \\
\text { lab } \\
(\mathrm{ag} / \mathrm{L} \\
\mathrm{as} \\
\mathrm{CaCo} 3)\end{array}$ \\
\hline
\end{tabular}

1981:

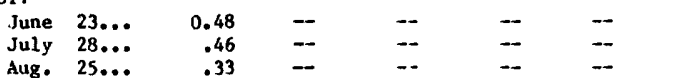

$\begin{array}{llllll}\text { Aug. } 25 . . . & .33 & -- & -.- & - & -\end{array}$

Sept. $23 \ldots . . . \quad .58 \quad 0.9$

$\begin{array}{llllll}\text { 0ct. } 28 . . . & .77 & - & - & - \\ & .23 & - & - & --\end{array}$

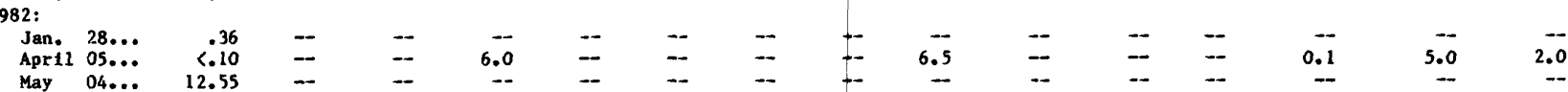

See footnote at end of table. 


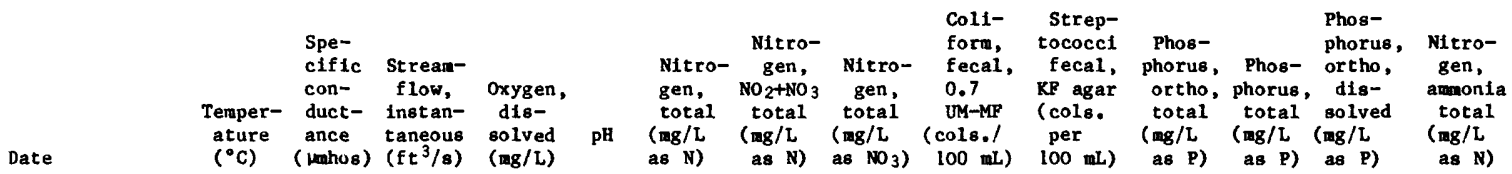

Station 014477.50, Baar Creek near White Haven, $\mathrm{Pa}$.

1981

\begin{tabular}{|c|c|c|c|c|c|c|c|c|c|c|c|c|c|c|c|}
\hline June & $23 . .$. & 21.0 & 52 & 70 & 9.0 & 5.0 & 0.37 & 0.02 & 1.6 & 56 & 80 & 0.004 & 0.028 & $<0.001$ & $\begin{array}{r}<0.010 \\
.020\end{array}$ \\
\hline July & $28 \ldots$ & 21.2 & 66 & 22 & 7.8 & 4.5 & .16 & .01 & .70 & ${ }^{1} \mathrm{~K} 15$ & 240 & .004 & .004 & $<.001$ & .020 \\
\hline $\begin{array}{l}\text { Sept. } \\
\text { Oct. }\end{array}$ & $\begin{array}{l}23 . . \\
20\end{array}$ & $\begin{array}{r}13.9 \\
5.9\end{array}$ & $\begin{array}{l}65 \\
39\end{array}$ & $\begin{array}{l}22 \\
19\end{array}$ & $\begin{array}{r}9.5 \\
10.7\end{array}$ & $\begin{array}{l}4.5 \\
4.3\end{array}$ & $\begin{array}{l}.42 \\
.37\end{array}$ & $\begin{array}{l}.04 \\
.05\end{array}$ & $\begin{array}{l}1.8 \\
1.6\end{array}$ & $41^{3}$ & $\begin{array}{l}36 \\
13\end{array}$ & $\begin{array}{l}.005 \\
.011\end{array}$ & $\begin{array}{l}.015 \\
.003\end{array}$ & $\begin{array}{l}.005 \\
.004\end{array}$ & $<.01$ \\
\hline $\begin{array}{l}\text { Jan. } \\
\text { April } \\
\text { May }\end{array}$ & $\begin{array}{l}28 \ldots . . \\
05 \ldots . \\
04 \ldots\end{array}$ & $\begin{array}{r}.2 \\
1.6 \\
13.2\end{array}$ & $\begin{array}{l}93 \\
50 \\
45\end{array}$ & $\begin{array}{r}67 \\
230 \\
56\end{array}$ & $\begin{array}{l}13.2 \\
14.6 \\
10.0\end{array}$ & $\begin{array}{l}4.8 \\
4.1 \\
4.4\end{array}$ & $\begin{array}{r}.45 \\
.45 \\
.72\end{array}$ & $\begin{array}{l}.14 \\
.03 \\
.02\end{array}$ & $\begin{array}{l}2.0 \\
2.0 \\
3.2\end{array}$ & $\overline{1}$ & $=$ & $\begin{array}{r}.010 \\
.008 \\
<.010\end{array}$ & $\begin{array}{l}.009 \\
.011 \\
.010\end{array}$ & $\begin{array}{r}.005 \\
.005 \\
<.010\end{array}$ & \\
\hline
\end{tabular}

\begin{tabular}{|c|c|c|c|c|c|c|c|c|c|c|c|c|c|c|}
\hline $\begin{array}{l}\text { Nitro- } \\
\text { gen, aw- } \\
\text { monia }+ \\
\text { organic } \\
\text { total } \\
(\mathrm{mg} / \mathrm{L} \\
\mathrm{ag} \mathrm{N})\end{array}$ & $\begin{array}{l}\text { Magne- } \\
\text { sium, } \\
\text { dis- } \\
\text { solved } \\
\text { ( } \mathrm{mg} / \mathrm{L} \\
\text { as Mg) }\end{array}$ & $\begin{array}{l}\text { Calcium } \\
\text { Dis- } \\
\text { solved } \\
\text { (mg/L } \\
\text { as Ca) }\end{array}$ & $\begin{array}{l}\text { Chlo- } \\
\text { ride, } \\
\text { dis- } \\
\text { solved } \\
\text { (mg/L } \\
\text { as Cl) }\end{array}$ & $\begin{array}{c}\text { Hard- } \\
\text { ness } \\
(\mathrm{ag} / \mathrm{L} \\
\mathrm{as} \\
\left.\mathrm{CaCO}_{3}\right)\end{array}$ & $\begin{array}{l}\text { Kanga- } \\
\text { nese, } \\
\text { dis- a } \\
\text { solvad } \\
\text { ( } \mu \mathrm{g} / \mathrm{L} \\
\text { as } \mathrm{Mn})\end{array}$ & $\begin{array}{l}\text { Sod1um } \\
\text { adsorp- } \\
\text { t1on } \\
\text { ratio }\end{array}$ & $\begin{array}{l}\text { Sodium, } \\
\text { dis- } \\
\text { Bolved } \\
(\mathrm{mg} / \mathrm{L} \\
\mathrm{as} \mathrm{Na})\end{array}$ & $\begin{array}{l}\text { Sulfate } \\
\text { dis- } \\
\text { solved } \\
(\mathrm{mg} / \mathrm{L} \\
\left.\mathrm{ag}_{8} \mathrm{SO}_{4}\right)\end{array}$ & $\begin{array}{l}\text { Iron, } \\
\text { dis- } \\
\text { solved } \\
\text { ( } \mathrm{ug} / \mathrm{L} \\
\mathrm{ag} / \mathrm{Fe})\end{array}$ & $\begin{array}{l}\text { Sedi- } \\
\text { ment, } \\
\text { dis- } \\
\text { charge, } \\
\text { sus- } \\
\text { pendad } \\
\text { (Ton/d) }\end{array}$ & $\begin{array}{l}\text { Sedi- } \\
\text { ment, } \\
\text { sus- } \\
\text { pened } \\
\text { (mg/L) }\end{array}$ & $\begin{array}{c}\text { Acidity } \\
(\mathrm{mg} / \mathrm{L} \\
\mathrm{as} \mathrm{H})\end{array}$ & $\begin{array}{c}\text { Actdity } \\
(\mathrm{mg} / \mathrm{L} \\
\mathrm{as} \\
\mathrm{CaCo} 3)\end{array}$ & $\begin{array}{c}\text { Alka- } \\
\text { Iinfty } \\
\text { lab } \\
\text { (mg/L } \\
\text { as } \\
\left.\mathrm{CaCo}_{3}\right)\end{array}$ \\
\hline
\end{tabular}

$1981:$

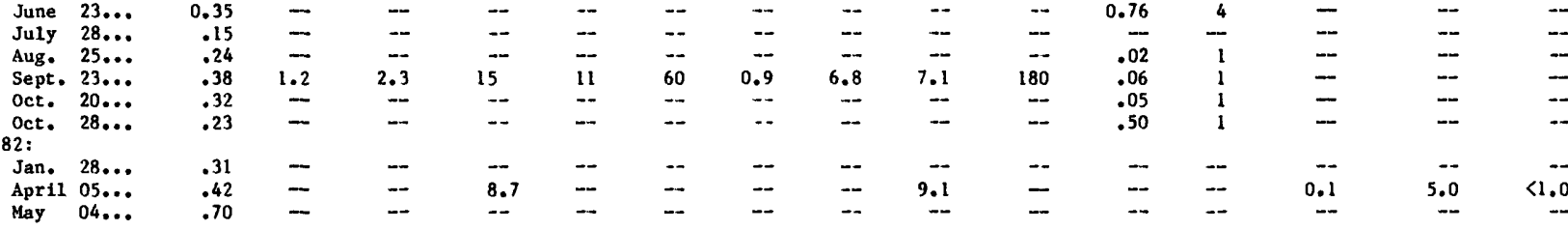

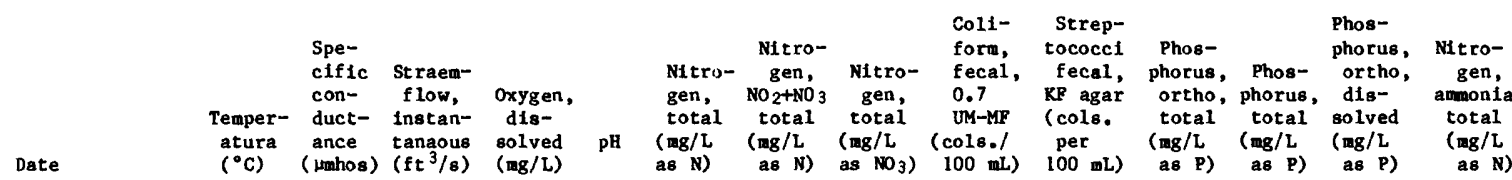

Station 014478.00 , Lehigh River below Francis $\mathrm{E}$. Walter Reservolr near White Haven, Pa.

1981:

\begin{tabular}{|c|c|c|c|c|c|c|c|c|c|c|c|c|c|c|c|}
\hline Juna & $23 . .$. & 17.0 & - & 786 & 10.0 & 5.7 & 0.52 & 0.02 & 2.3 & - & -- & $<0.001$ & 0.021 & $<0.001$ & 0.240 \\
\hline $\begin{array}{l}\text { July } \\
\text { Aug. }\end{array}$ & $\begin{array}{l}28 \ldots \\
25 \ldots\end{array}$ & $\begin{array}{l}19.0 \\
19.8\end{array}$ & $\begin{array}{l}42 \\
44\end{array}$ & $\begin{array}{r}172 \\
41\end{array}$ & $\begin{array}{l}8.7 \\
\mathbf{8 . 6}\end{array}$ & $\begin{array}{l}5.4 \\
5.6\end{array}$ & $\begin{array}{r}.49 \\
.58\end{array}$ & $\begin{array}{l}.01 \\
.07\end{array}$ & $\begin{array}{l}2.1 \\
2.6\end{array}$ & $\overline{-}$ & $=$ & $\begin{array}{r}.017 \\
.002\end{array}$ & $\begin{array}{r}.028 \\
.017\end{array}$ & .007 & $\begin{array}{r}.200 \\
.020\end{array}$ \\
\hline $\begin{array}{l}\text { Sept. } \\
\text { Oct. }\end{array}$ & $\begin{array}{l}23 . \ldots \\
20 \ldots\end{array}$ & $\begin{array}{l}15.6 \\
12.1\end{array}$ & $\begin{array}{l}41 \\
34\end{array}$ & $\begin{array}{r}44 \\
182\end{array}$ & $\begin{array}{r}9.1 \\
10.3\end{array}$ & $\begin{array}{l}5.8 \\
5.5\end{array}$ & $\begin{array}{r}.56 \\
.93\end{array}$ & .08 & $\begin{array}{l}2.5 \\
4.1\end{array}$ & $\overline{-}$ & -- & $\begin{array}{l}.002 \\
.005 \\
.005\end{array}$ & $\begin{array}{l}.023 \\
.009\end{array}$ & $\begin{array}{l}.005 \\
.005\end{array}$ & $\begin{array}{l}.060 \\
.070\end{array}$ \\
\hline $\begin{array}{l}\text { April } \\
\text { May }\end{array}$ & $\begin{array}{l}05 . . \\
04 \ldots\end{array}$ & $\begin{array}{r}3.9 \\
11.4\end{array}$ & $\begin{array}{l}35 \\
24\end{array}$ & $\begin{array}{r}2710 \\
502\end{array}$ & $\begin{array}{r}13.6 \\
4.6\end{array}$ & $\begin{array}{l}5.3 \\
5.6\end{array}$ & .64 & $\begin{array}{r}.15 \\
<.09\end{array}$ & 2.8 & $\overline{1}$ & $\overline{23}$ & $\begin{array}{r}.009 \\
<.010\end{array}$ & $\begin{array}{l}.011 \\
.011\end{array}$ & $<.006$ & $\begin{array}{l}.060 \\
.030\end{array}$ \\
\hline
\end{tabular}

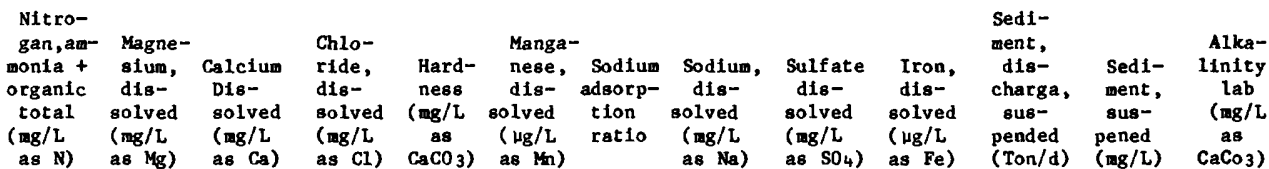

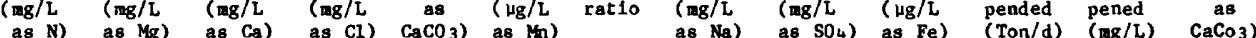

1981

\begin{tabular}{|c|c|c|c|c|c|c|c|c|c|c|c|c|c|c|}
\hline June & $23 . \ldots$ & 0.50 & - & -- & $\cdots$ & - & - & -- & - & - & -- & - & - & - \\
\hline $\begin{array}{l}\text { Oct. } \\
\text { 982: }\end{array}$ & $20 \ldots$ & .88 & - & - & - & - & - & - & - & - & - & - & - & - \\
\hline April & $05 .$. & .49 & - & - & 6.8 & - & - & $\cdots$ & - & 7.4 & - & - & -- & 3.0 \\
\hline May & $04 .$. & .72 & - & -- & - & - & - & - & - & - & - & - & - & \\
\hline
\end{tabular}

1 Best estimated based upon nonideal counting conditions. 


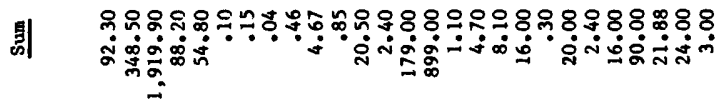

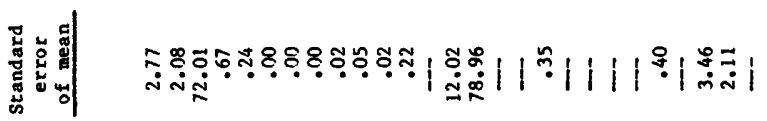

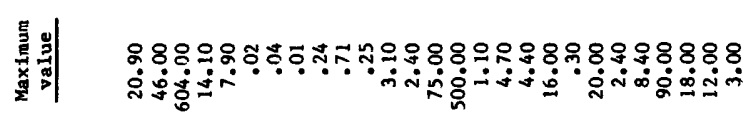

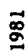

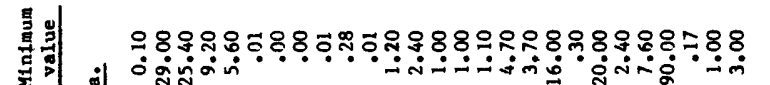
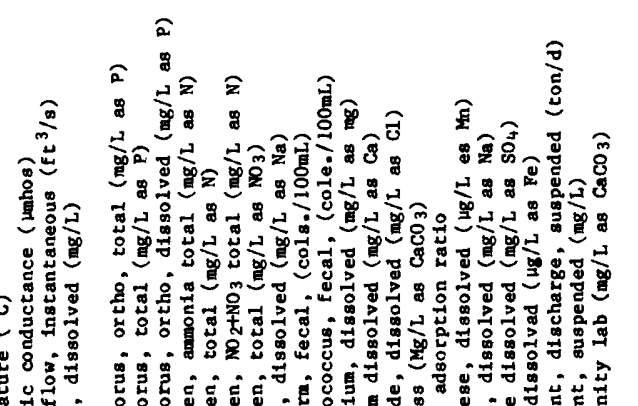

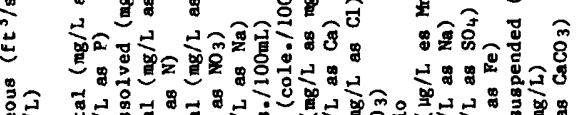

落 0

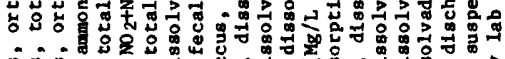

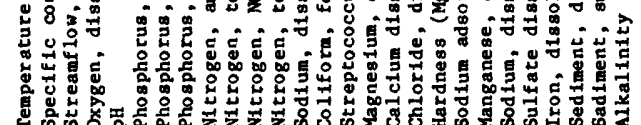

岕|

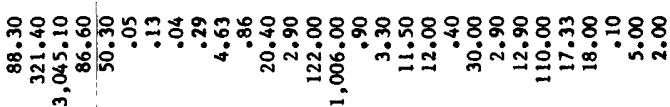

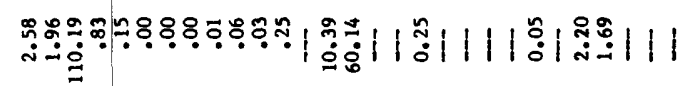

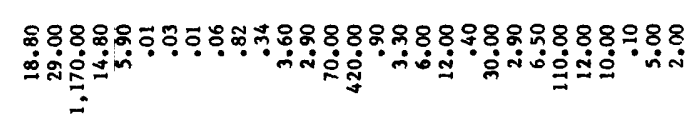

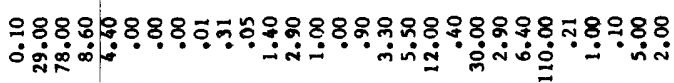

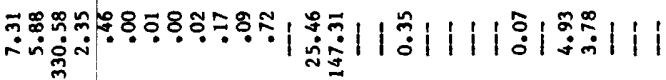

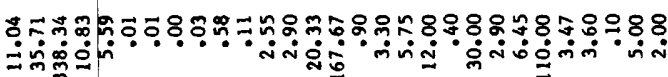

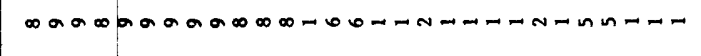

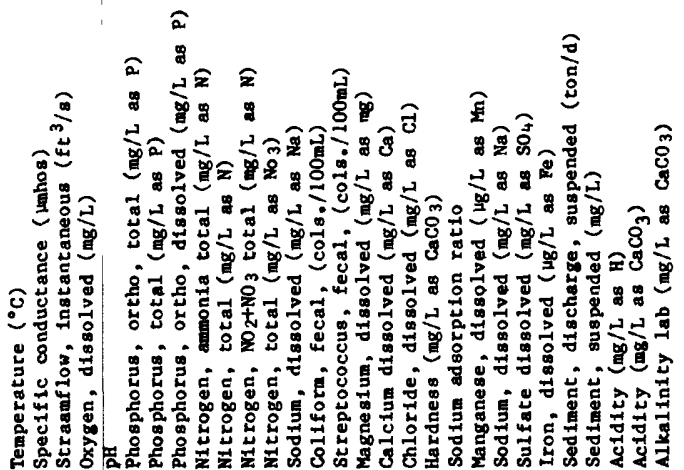

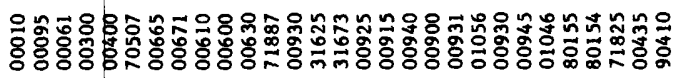




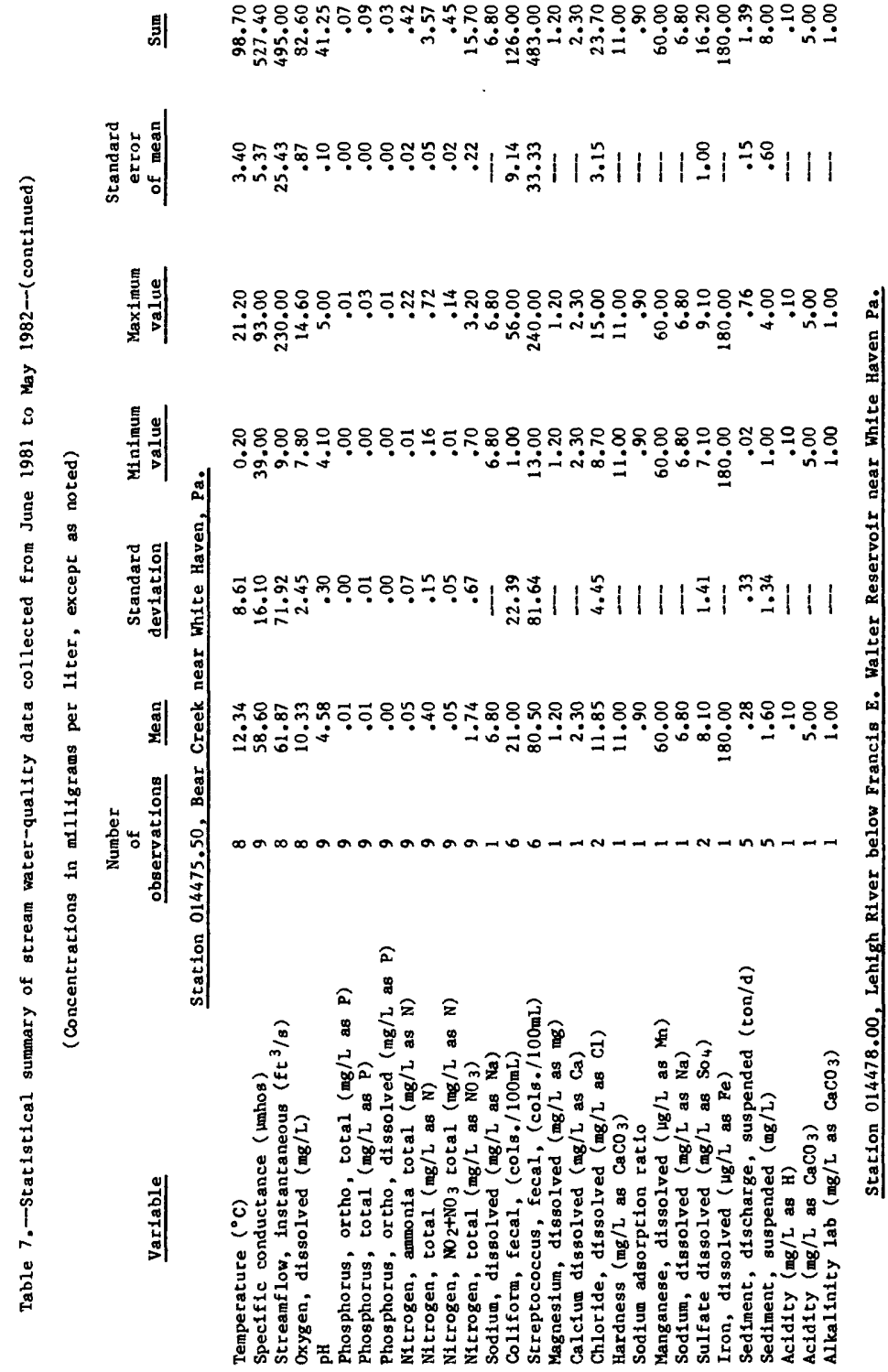

It

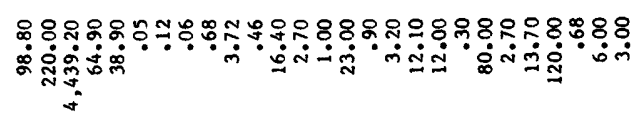

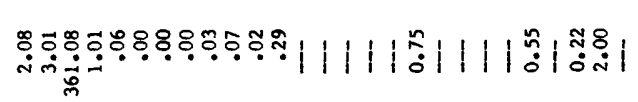

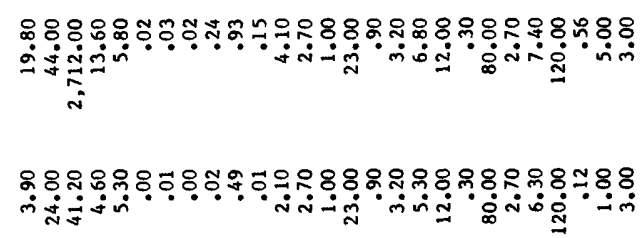

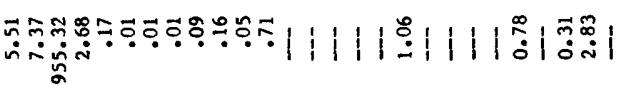

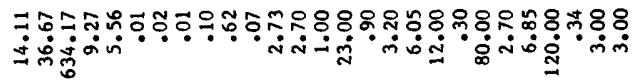

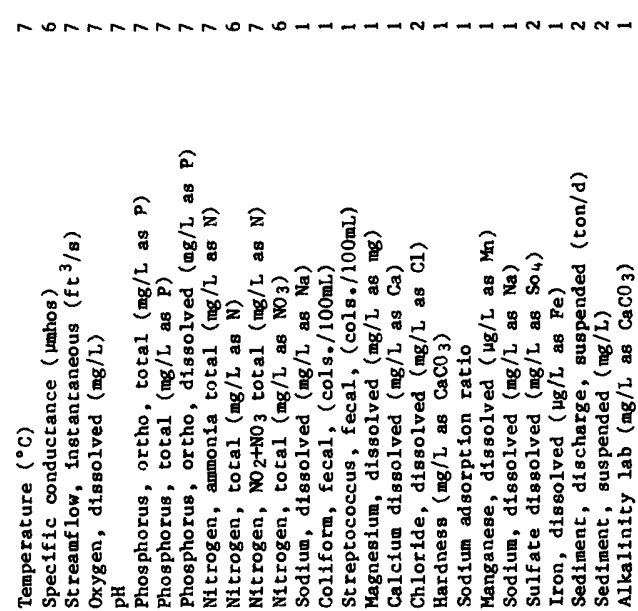

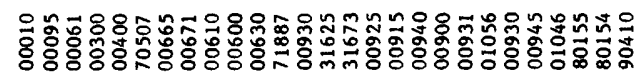




\section{Temperature}

Water temperature is a controlling factor in the biological characteristics of a stream and influences its physical and chemical regime. Pennsylvania Water Quality Standards (1979) stipulate that for the Lehigh River from its source to Route 903 bridge at Jim Thorpe (fig. 2) there shall be no rise when ambient temperature is $58^{\circ} \mathrm{F}$ or above; not more than $5^{\circ} \mathrm{F}$ rise above ambient temperature until the stream reaches $58^{\circ} \mathrm{F}$ and shall not be changed by more than $2^{\circ} \mathrm{F}$ during any 1-hour period. Temperatures of all waters within the watershed are curcently meeting this water-quality criteria. Maximum stream temperatures recorded were $70^{\circ} \mathrm{F}\left(21^{\circ} \mathrm{C}\right)$. There are no heated effluents discharging into the streams.

\section{Dissolved Oxygen}

Dissolved-oxygen concentrations are perhaps the single most important criterion in determining the health of a stream. It is essential that dissolvedoxygen concentrations be maintained above $5.0 \mathrm{mg} / \mathrm{L}$ for a viable cold-water Fishery. Pennsylvania Water Quality Standards (1979) for streams in the Upper Lehigh River basin stipulate that dissolved oxygen shall not be less than $7.0 \mathrm{mg} / \mathrm{L}$ at any time.

Dissolved-oxygen values determined in conjunction with this study indicate that this criterion is being met most of the time. A check of the biweekly and monthly historical data, collected since 1975, indicates that waters of the Lehigh River and Tobyhanna Creek had dissolved-oxygen concentrations of less than $7.0 \mathrm{mg} / \mathrm{L}, 4$ and 5 percent of the time, respectively; but were never less than $5.0 \mathrm{mg} / \mathrm{L}$. Bear Creek had no values less than $7.0 \mathrm{mg} / \mathrm{L}$.

\section{Value of $\mathrm{pH}$}

The term $\mathrm{pH}$ is a universal way of expressing the acidity or alkalinity of a solution. More precisely, it is a way of expressing the hydrogen-ion activity. A pH of 7.0 is neutral; greater than 7.0 is considered alkaline and less than 7.0 is considered acidic.

The streams draining into the Francis $E$. Walter Reservoir are typtcal of those draining the Appalachian Plateau, with pH values generally ranging from 5.5 to 7.0. The waters of Bear Creek are somewhat more acidic than those of either the Lehigh River or Tobyhanna Creek with pH values normally in the range of 4.3 to 5.0 . The acidic waters are believed the result of acidic precipitation ( $\mathrm{pH} 4.3$ ), high concentrations of carbonic and other organic acids that are products of decaying plant matter, and poor buffering capacity of basin soils.

Pennsylvania Water Quality Standards (1979) for the Lehigh River and tributarles from its source to the Francis $\mathrm{E}$. Walter Reservolr, stipulate that the $\mathrm{pH}$ shall be not less than 6.0 and not more than 9.0. Based upon the 1975 to 1981 data, the minimum $\mathrm{pH}$ of 6.0 is not being met about 12 percent of the time in the Lehigh River and more frequently in the other tributaries. There is some evidence, as reported in the 1980 Corps of Engineers report number RCS-DAEN-CWE-15, that precipitation results in a decrease in stream pH. A decrease is to be expected because any flushing of the forest and bogs would tend to release waters high in organic acids. 
Specific Conductance and Dissolved Solids

Specific conductance is a measure of the ionic strength and ability of a solution to carry an electrical current. Dissolved solids are the anhydrous residues of the dissolved substances in water.

Freestone streams draining the Appalachian Plateau are termed soft waters because they have specific conductances ranging from 22 to 57 micromhos and dissolved solids ranging from 14 to $40 \mathrm{mg} / \mathrm{L}$. (McCarren and Keighton, 1969). The waters of Bear Creek appear to be slightly higher in dissolved solids than either the Lehigh River at Stoddartsville or Tobyhanna Creek near Blakeslee. Reasons for this are not clear but may be related to differences in soils and geology.

Specific conductance and dissolved solids normally tend to follow the increases and decreases in discharge because of the availability of dissolved matter. As noted in previous Corps of Engineers water-qualtty-data reports 1978 to 1980 , the normal relation ( $D S=0.66 \pm 0.09 \mathrm{~K}$ ) between dissolved solids and specific conductance is not found. In fact, a regression analysis of 32 data pairs collected at station 014477.50 from 1978 to 1980 failed to show a good correlation. The reason for the poor correlation is not clear but appears to be related to the retention and delayed flushing of highly organic bog material.

\section{Anions and Cations}

Dissolved sodium, calcium, and magnesium were analyzed from each of the major tributaries. Although cations were not routinely quantified as part of this water-quality study, they may be useful for determining future land/use and water-quality relationships. Dissolved chloride and sulfate are likewise useful in catagorizing water types and land-use changes. Historical water analyses published by McCarren and Keighton (1969) and samples collected on September 23, 1981 and April 5, 1982 show that the dissolved solids in waters tributary to the impoundment are comprised predominantly of calctum and sulfate ions during higher flows and calclum and bicarbonate inns during base flow. Judglng by some concentrations dating back to 1944 (McCarren and Keighton, 1969), there have been no significant changes in water chemistry to date (1982) that have affected the total dissolved-solids content.

\section{Nitrogen}

Principal sources of nitrogen to the reservoir ecosystem are forest runoff, swamp and marsh runoff, precipitation on water surface, septic tank runoff, leaves, dust, pollen, waterfowl waste, and fixation by bacteria and blue-green aljae.

Forms of aitrogen examined were total nitrite plus nitrate, total ammonia, total ammonia plus organic nitrogen, and total nitrogen. Standards for the upper Lehigh River basin require that nitrite plus nitrate nitrogen not exceed 10 $\mathrm{mg} / \mathrm{s}$ (Pennsyvlania Water Quality Standards, 1979). U. S. Environmental Protection Agency (1976) criterion for ammonia $\left(\mathrm{NH}_{3}+\mathrm{NH}_{4}\right)$ is $0.02 \mathrm{mg} / \mathrm{L}$ as unionized amonia. Due to the equiltbrium of dissolved ammonia in water the concentrations of total ammonia in the streams tributary to the Francis E. Walter 
Reservoir would have to exceed $3.5 \mathrm{mg} / \mathrm{L}$ before the criterion would be exceeded. As evidenced by the maximum total ammonia values of $0.24 \mathrm{mg} / \mathrm{L}$, it is unlikely that any un-ionized ammonia exists in the upper Lehigh River drainage. Nitrite plus nitrate concentrations were also well below the state criterta.

The long-term annual averages of total nitrogen loads transported by tributarles to Francis E. Walter Reservoir and outlet stream are estimated in table 8. The total nitrogen inflow froin tributary sources is estimated to be 238 tons per year or 0.82 tons per $\mathrm{mi}^{2}$. Total nitrogen transport curves for the three tributaries and the outflow are shown in figure 6.

\section{Phosphorus}

Potential sources of phosphorus to the reservoir include agricultural runoff, swamps and forest runoff, domestic waste, and septic-tank discharge. other sources are direct precipitation on the water surface, dry fallout such as leaves, dust, seeds, and pollen; groundwater influxes, sediment recycling, and aquatic bird and aninal wastes.

Species of phosphorus examined were dissolved orthophosphate, total orthophosphate, and total phosphorus. Due to the anticipated low levels of phosphorus present, all analyses were done at a sensitivity level of $0.001 \mathrm{ng} / \mathrm{L}$. While the 1979 Pennsylvania Water-Quality Standarls do not have a criterion for phosphorus, the 1976 Enviroumental Protection Agency Quality Criteria for Water suggests that "to prevent the development of biological nuisances and to control accelerated or cultural eutrophication, total phosphates or phosphorus ( $P$ ) should not exceed $50 \mu \mathrm{g} / \mathrm{L}$ in any stream at the point where it enters any lake or reservoir."

The tributary samples collected from June 1981 to May 1982 indicate the Environnental Protection Agency criterion is not exceeded, even during runoff events. Sonewhat higher concentrations were found in the lehigh River at Stoddartsville than at other sires. The range and mean values found at each stream station are given in the statistical summary in table 7.

The most biologically active form of phosphorus is dissolved orthophosphate. The soluable orthophosphate is readily taken up by green plants, and therefore, is most important for the growth of algal cells. The concentrations of dissolved orthophosphate phosphorus ranged from 0.000 to $0.007 \mathrm{mg} / \mathrm{L}$.

Phosphorus loading fron the major tributaries was calculated by the flowduration method in the same manner as total nitrogen. The long-term annual. averages of total phosphorus transported by tributaries to Francis E. Walter Reservoir and outlet stream are estimated in table 9. The total phosphorus inflow from gaged and ungaged trlbutarles is estimated to be 10 tons per year, i) 0.03 tons per ni $^{2}$. Slightly higher loads at the outflows are attributed to) higher concentration in the hypolimetic water. Total phosphorus transport curves for the three tributarles and the outflow are shown in figure 6 . 
Table 8.--Long-term annual averages of total nitrogen transported by tributaries to Francis E. Walter Reservoir and from reservoir to Lehigh River

(Data in short tons per year)

Tributary inflow

\begin{tabular}{|c|c|c|c|c|}
\hline $\begin{array}{c}\begin{array}{c}\text { Tobyhanna } \\
\text { Creek }\end{array} \\
\text { (Sta } 014477.20) \\
\end{array}$ & $\begin{array}{r}\text { Lehigh River } \\
\text { (Sta 014475.00) } \\
\end{array}$ & $\begin{array}{c}\text { Bear Creek } \\
\text { (Sta } 014477.50) \\
\end{array}$ & Ungaged & $\begin{array}{l}\text { Lehigh River } \\
\text { below reservoir } \\
\text { (Sta 014478.00) }\end{array}$ \\
\hline 109.5 & 75.6 & 18 & 35 & 284.7 \\
\hline
\end{tabular}

Total inflow $=238$ tons per year. 


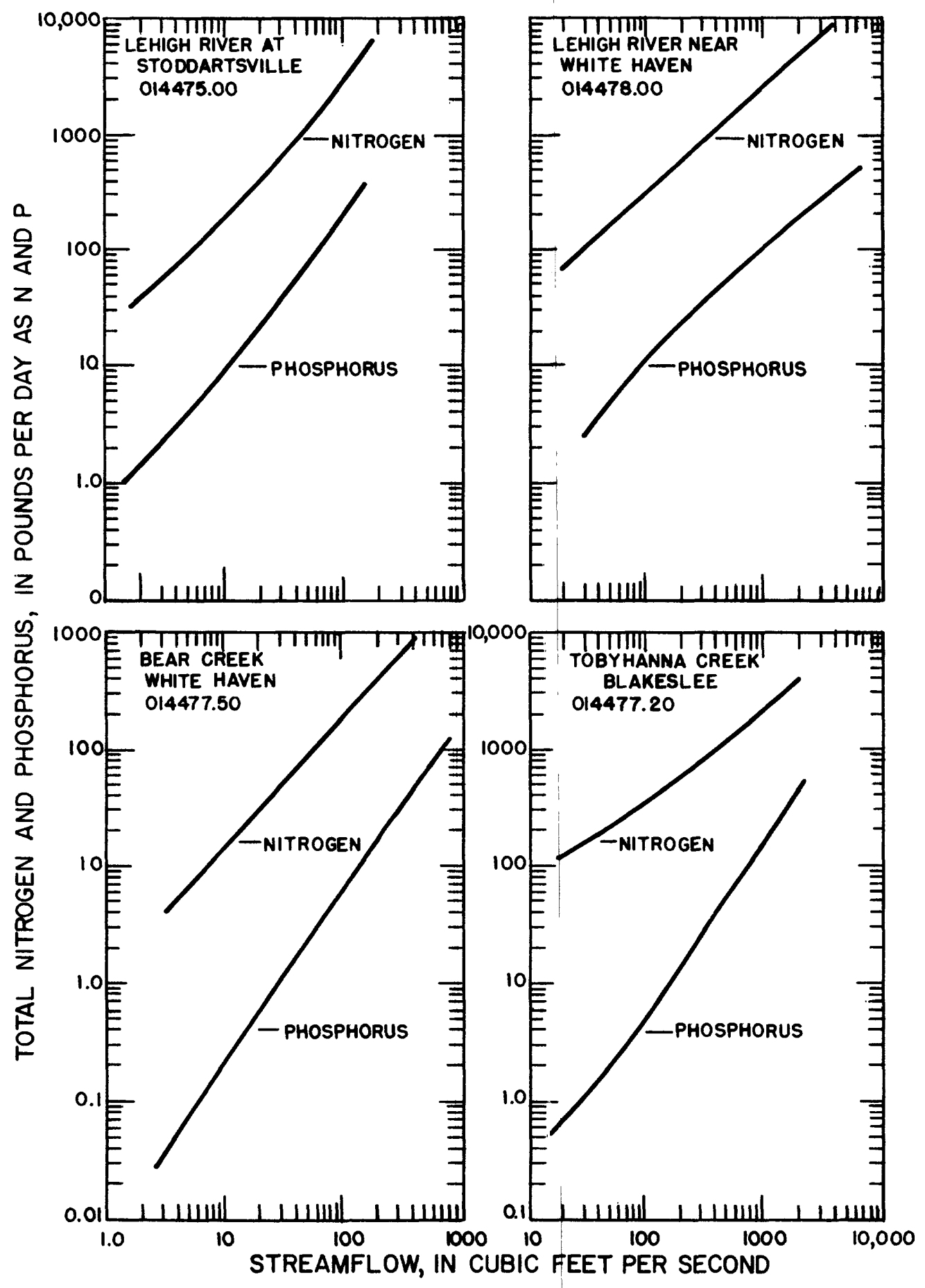

Figure 6.--Nitrogen and phosphorus transport curves for tributary streams. 
Table 9.--Long-term annual averages of total phosphorus transported by tributarles to Francis E. Walter Reservoir and from reservoir to Lehigh River

(Data in short tons per year)

\begin{tabular}{ccccc}
\hline \multicolumn{5}{c}{ Tributary inflow } \\
\hline $\begin{array}{c}\text { Tobyhanna } \\
\text { Creek }\end{array}$ & Lehigh River & Bear Creek & Ungaged & $\begin{array}{c}\text { Lehigh River } \\
\text { below reservoir } \\
\text { (Sta 014478.00) }\end{array}$ \\
\hline 3.6 & 4.2 & 0.8 & 1.6 & 11.7 \\
\hline
\end{tabular}

Total inflow $=10.0$ tons per year. 
Trace Metals

Trace metals in the outflow water were analyzed because they are representative of the hypolimnetic discharge water and would be of interest to downstream water users. An anaerobic hypolimnion tends to contain the highest concentrations of metals due to chemical reduction. Analyses were done for the dissolved metals - barium, copper, lead, manganese, zinc, cadmium, selenium, arsenic, silver, magnesium, iron, and mercury so that comparisons with stream standards could be made. These data are summarized in table 10. There is no indication, based upon this sample, that any of the metals presently exceed or even approach stream standards (Pennsylvania Water Quality Standards, 1979).

\section{Bacteria}

The use of fecal coliform and fecal streptococcus bacteria as indicators of the possible presence of disease-causing organisms has been standard practice in water microbiology for many years and is the basis of the Pennsylvania Sanitary Water Board (1979) water-quality criterion which states that "during the swimming season (May 1 through September 30) the fecal coliform level shall not exceed a geometric mean of 200 per $100 \mathrm{~mL}$ based upon five consecutive samples, each sample collected on different days; for the remainder of the year, the fecal coliform level shall not exceed a geometric mean of 2,000 per $100 \mathrm{~mL}$, based upon five consecutive samples collected on different days."

Fecal coliforms are a group of bacteria specific to the gut of warm-blooded animals. Therefore, their presence in water is a direct indication of fecal contamination. Unfortunately, the fecal coliform test does not distinguish between organisms that originate from humans from those of other warm-blooded animals. Studies by Geldreich and Kenner (1969) demonstrated that fecal coliform are at least four times more numerous than fecal streptococci in the feces of man.

Due to the scope of the sampling program, an insufficent number of samples was examined to calculate geometric means as prescribed in the water-quality criteria. However, enough samples were examined over the 12-month period at various hydrologic and climactic conditions to indicate the range in indicator bacterial densities. Results of the bacterial examination are summarized in table 11. Fecal coliform densities remained well below the levels considered hazardous to water contact forms of recreation and at no time did fecal coliform/fecal streptococcus ratios suggest human wastes were present in the streams.

\section{Suspended Sediment}

Fluvial sedimentation is the process of erosion, transport and deposition of soll by streams. The concentration of suspended sediment in a stream at a given time depends on the season of the year, land use, antecedent moisture conditions, and the nature of the precipitation intensity and pattern on the basin.

Concentrations of suspended sediment in the tributaries to Francis E. Walter Reservoir ranged from less that $1 \mathrm{mg} / \mathrm{L}$ at base flow to about $20 \mathrm{mg} / \mathrm{L}$ during storm runoff. Concentrations were low, due to the heavily forested soils and because the sandstones and glacial surface material have little fine material to be washed into the stream. 
Table 10.--Summary of dissolved trace-metal analyses at station 014478.00 Lehigh River near White Haven, September 23, 1981

(Concentrations in micrograns per liter)

Element

Barium

Copper

Lead

Manganese

Zinc

Cadmium

Selentum

Arsentc

Silver

Iron

Mercury

Magnesium

\section{Concentration}

100

1

1

80

10

$<1$

$<1$

2

$<1$

120

.2 


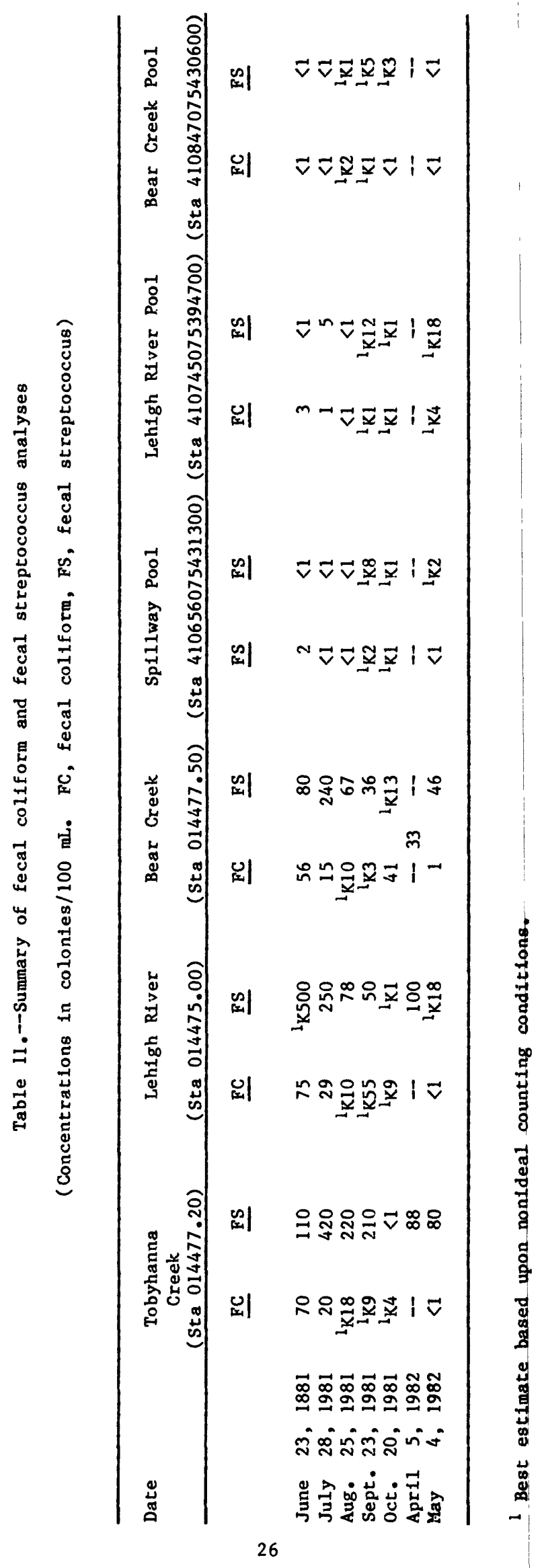


Sediment-transport loads are correspondingly low, ranging from less than 1 -ton per day in all tributaries to estimated maximum 10 ads of 100 , 150, and 400 tons per day at Lehigh River, Bear Creek, and Tobyhanna Creek, respectively (fig. 7). Annual yields are $5.7,12$, and 12 tons per square mile for the Lehigh River, Bear Creek, and Tobyhanna Creek, respectively. At these annual yields, the reservoir has a sedimentation rate of 5.7 acre-feet per year. This rate may be somewhat below the long-term average due to the below-normal precipitation during the study period.

\section{LAND USE}

Land use in the Francis E. Walter Dam watershed has been classified into five Geological Survey level I categories (Anderson and others, 1976). These include urban or built-up land, agricultural land, forest land, water, and wetland. The most recent (1973 and 1969) photo-revised $71 / 2$ feet Geological Survey topographic series maps were used to estimate land use on each of the three major tributaries.

For classification purposes, urban or built-up land comprises areas of intensive use with much of the land covered by structures. Included are villages, housing developments, strip development along highways, shopping centers, commercial complexes, a winter sports area, and a military reservation. Agricultural land includes cropland, pasture, and orchards. Forest land and water are self-explanatory. Wetlands are those areas where the water table is at, near, or above the land surface for a significant part of most years.

Land uses for each of the stream watersheds are shown in table 12. Forest land is easily the predominant type in each of the subbasins as it comprises from 63 to 90 percent of the land use and 75 percent of the entire reservoir watershed. Another interesting feature, and one which has a strong influence on the hydrology of the watershed, is the large percentage of land (13 percent) that is either under water or wetlands. The Tobyhanna, Creek subbasin contains the most urban or built-up land, approximately $18.8, \mathrm{mi}^{2}$ or 15 percent while the Bear Creek sub-basin contains the least with $2.3 \mathrm{mi}^{2}$ or 4 percent.

\section{LIMNOLOGY OF FRANCIS E. WALTER RESERVOIR}

\section{Hydrology}

Normal pool elevation for the reservoir is maintained at about 1,300 feet above sea level. Because of below normal precipitation in 1980 and 1981 , and the impending drought condition throughout the Delaware River basin, the pool elevation was raised to about 1,392 feet and maintained there until April 1982 when the drought condition was relieved by above normal rainfall.

The additional impounded water had the effect of drowning approximately 600 acres of forest and storing an additional 34,000 acre-feet of water. Some of the effects of this inundation will be dicussed in a following section.

The limnology of Francis E. Walter Reservoir includes a discussion of selected chemical, physical, and biological characteristics of the reservoir system that describe the trophic state, the physical setting, and the general quality of the water. The results of the current chemical analysis are reported in table 13. A statistical summary of the data is presented in table 14. 


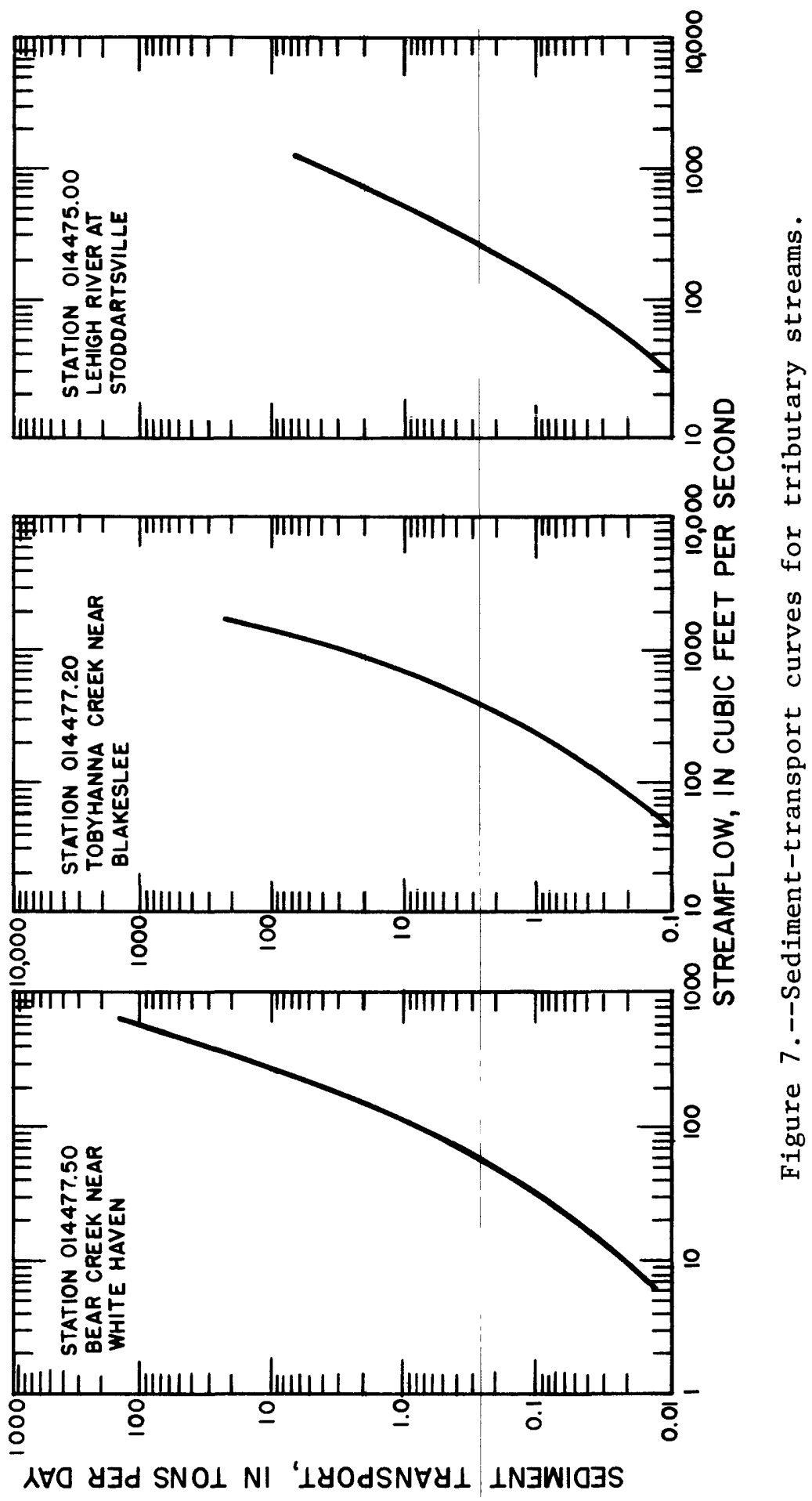




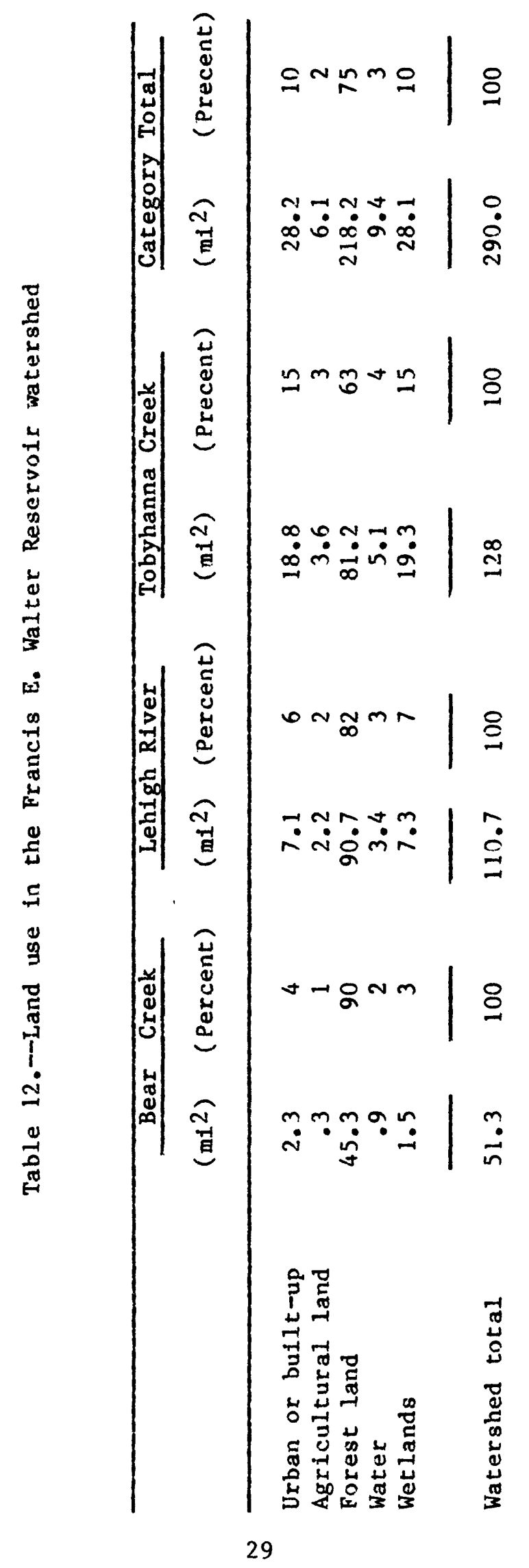




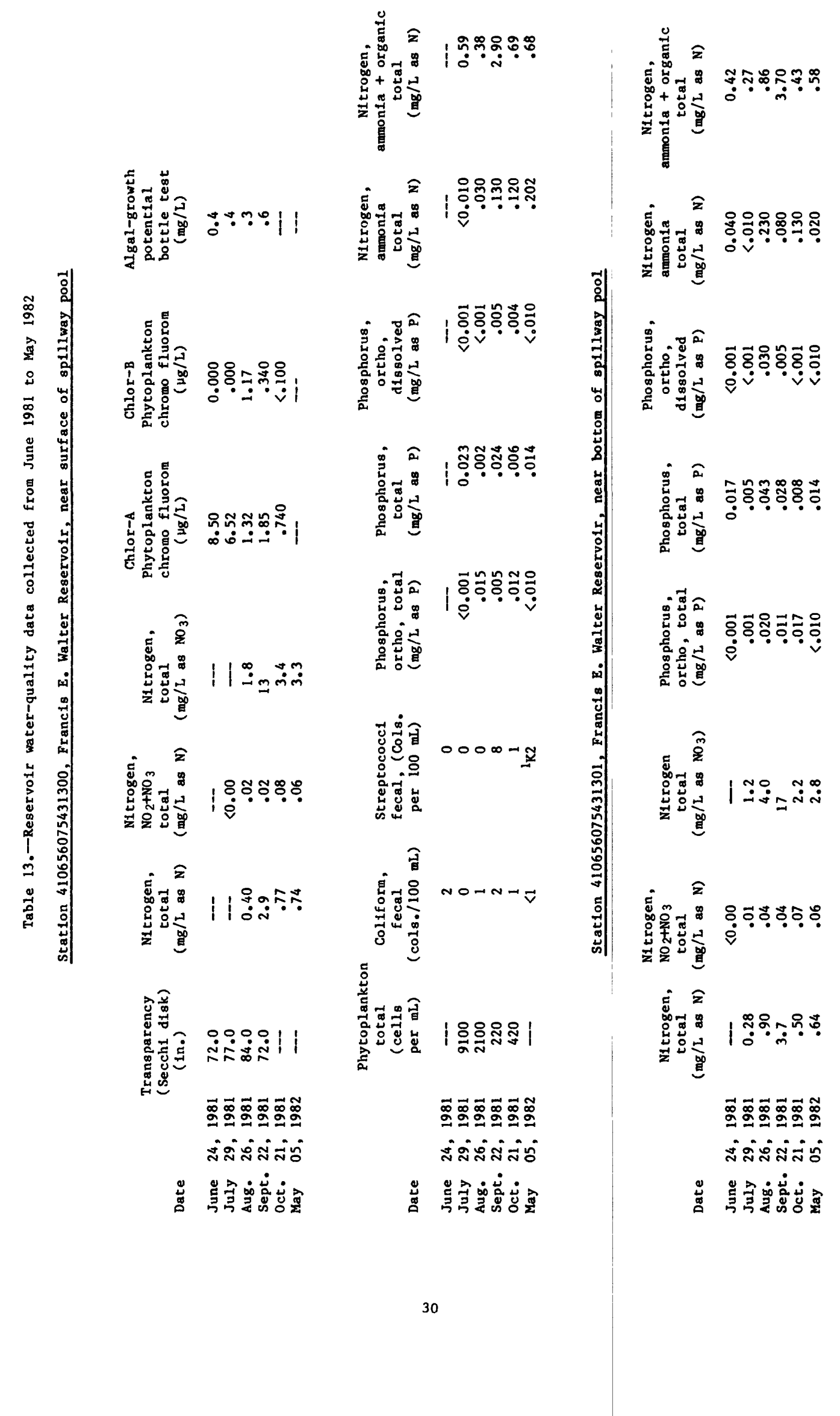




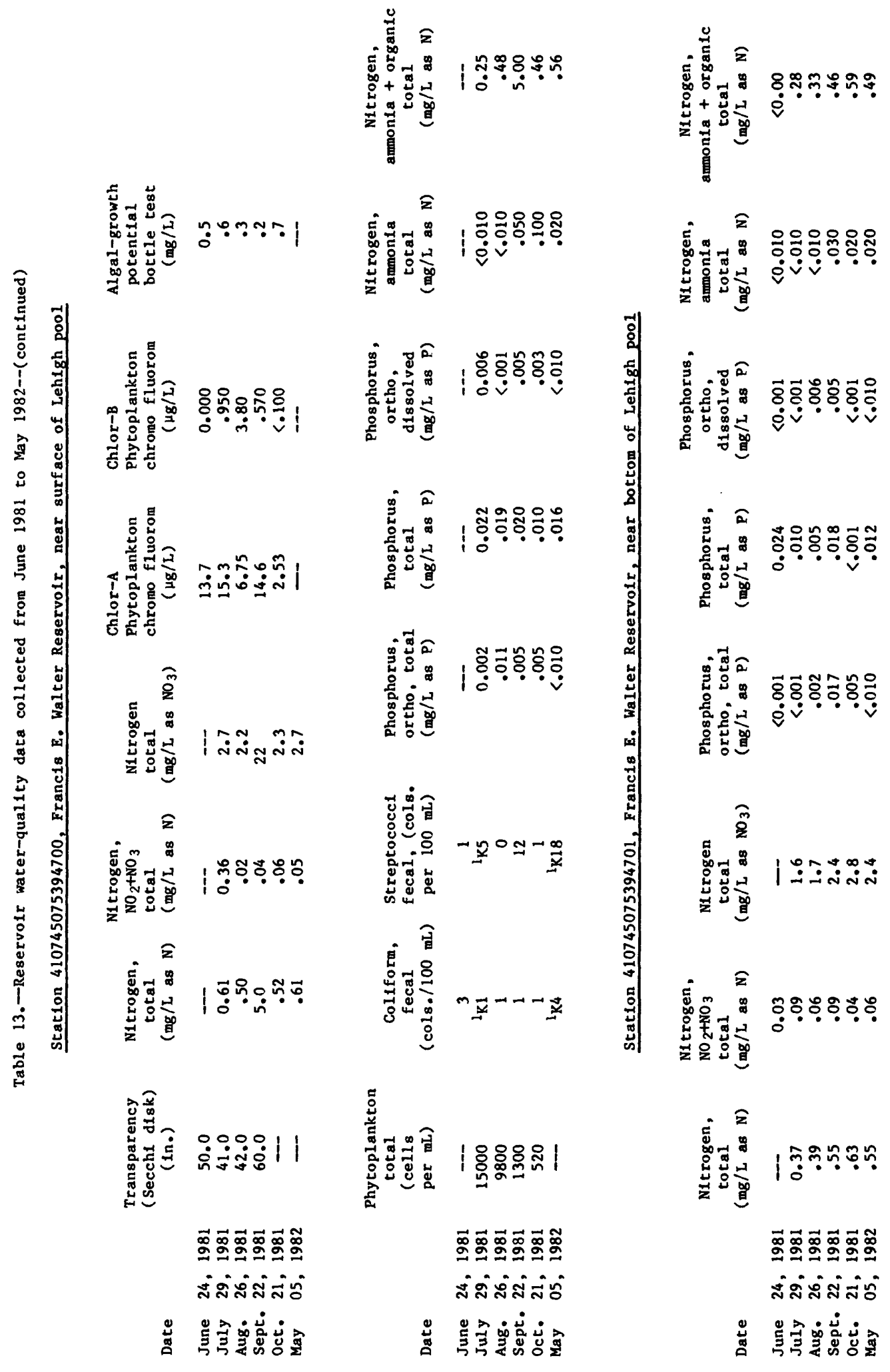




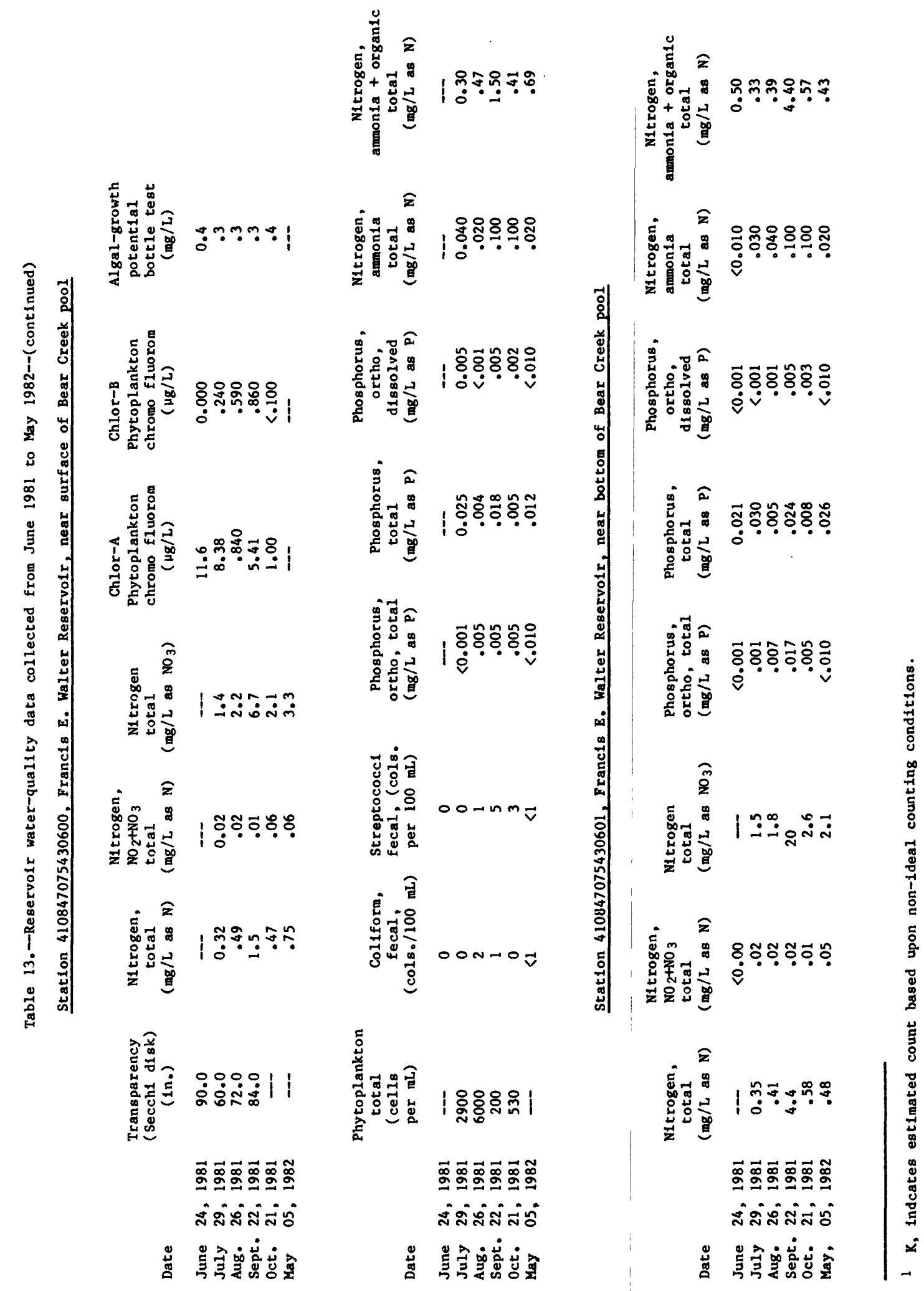




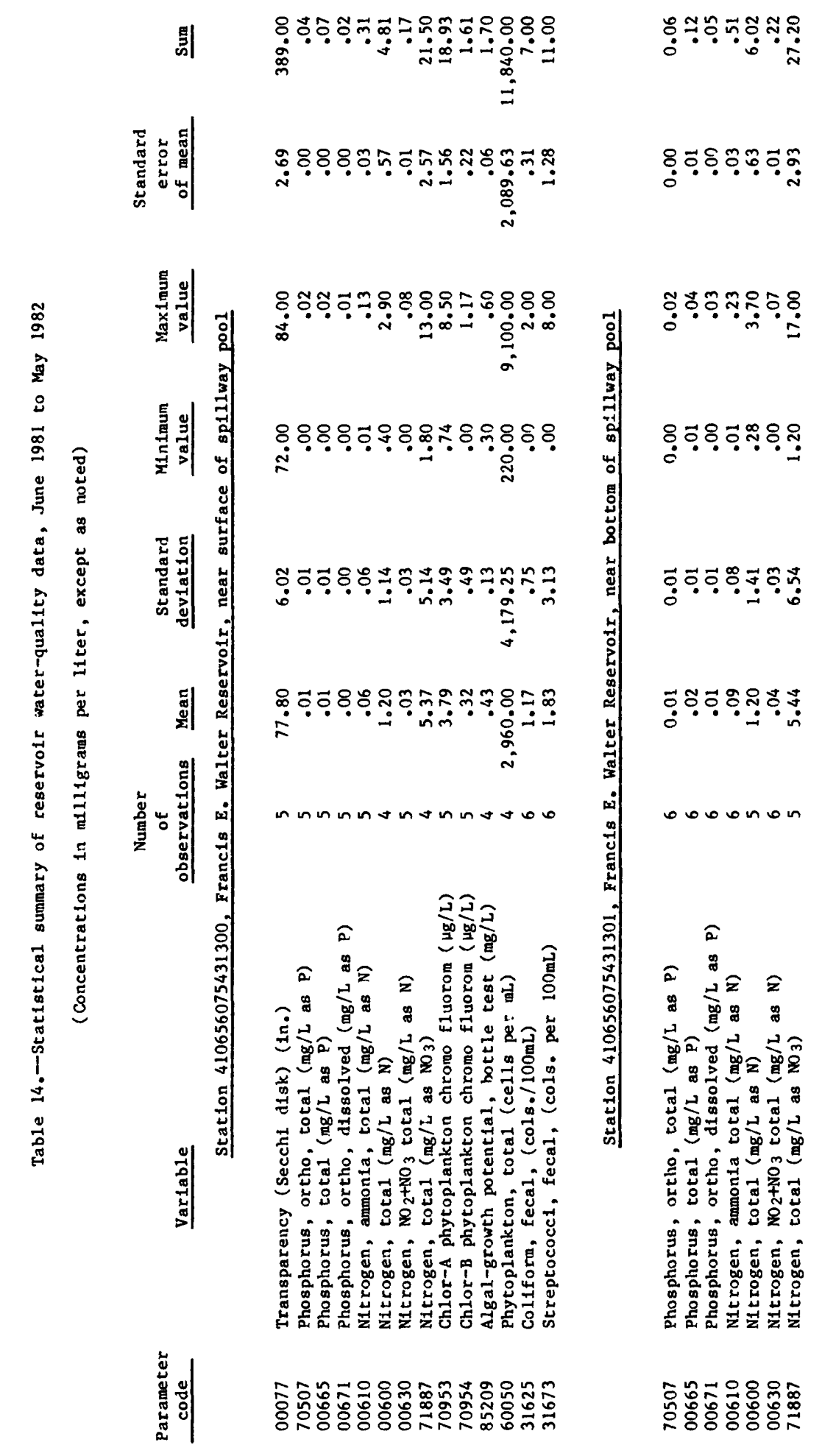




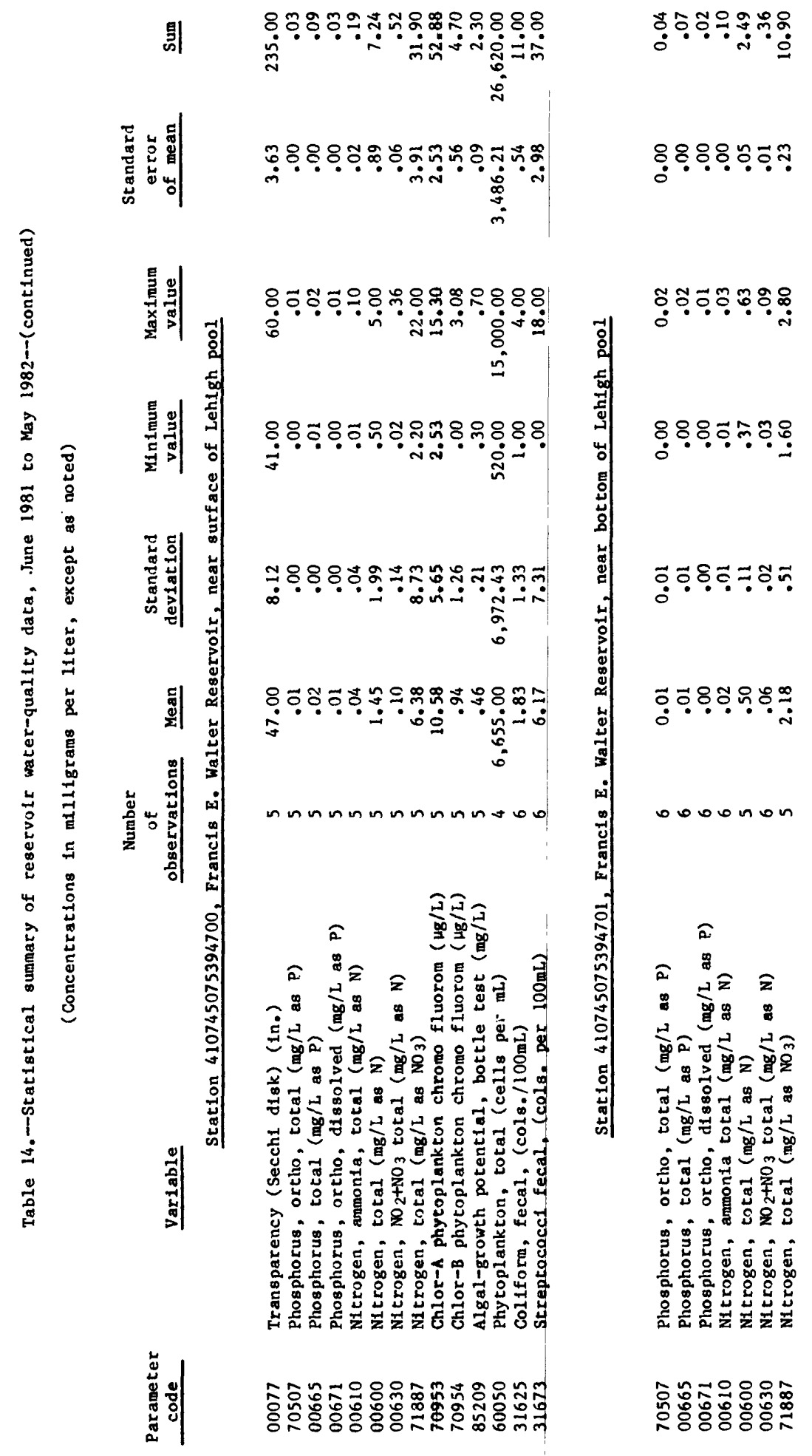




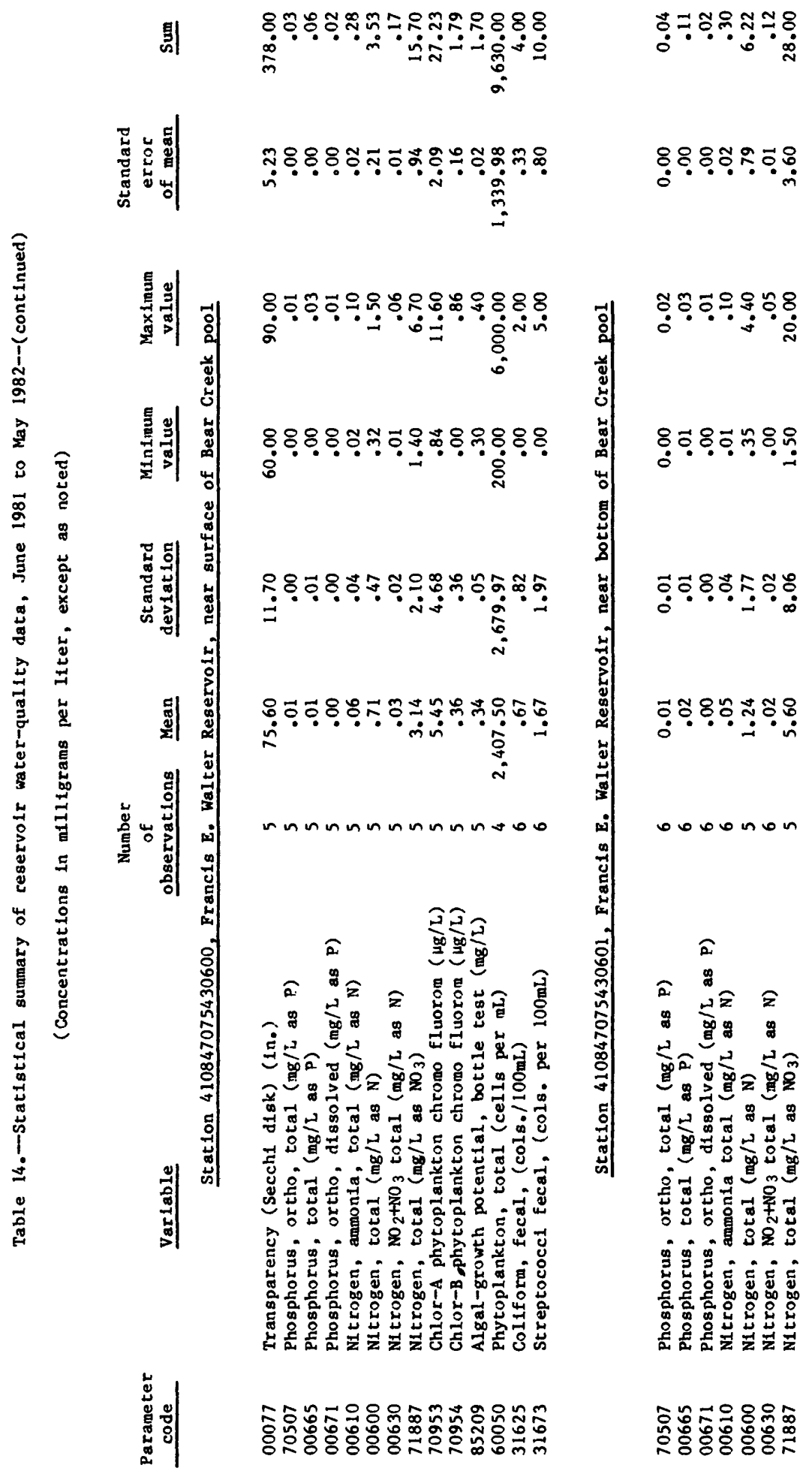


Complete profile data, collected at 5-foot intervals, for temperature, dissolved oxygen, $\mathrm{pH}$, and specific conductance at six locations, is on file at the U.S. Geological Survey, Harrisburg, and the U.S. Army Corps of Engineers, Philadelphia.

\section{Physical Characteristics}

\section{Temperature and Dissolved Oxygen}

Lakes and reservoirs within the temperate zone generally have temperatures of about $4^{\circ} \mathrm{C}$ throughout the water column at the tine of ice breakup each spring, and are subject to complete mixing by the wind. As air temperatures rise, the upper layers of water are warned. This process continues until the temperature-density gradient is sufficiently developed to produce a thermocline, and the so-called "summer stagnation period" begins. During this period the upper layer (the epilimnion) is separated from the lower layer (the hypolimnion) by a sharp temperature gradient (the thernocline).

When ale terneratures cool in the autimn, the temperature of the epilimnion decreases until it is the same temperature as the hypolimnion. The impoundment then enters the "autumn overturn" and is once more subjected to complete mixing by the wind. This process of heating and cooling is repeated each spring and autumn.

Plots of equal temperature for the deep water sampling station are shown in figure 8. Temperatures in the water column are nearly uniform except for a 2-week period in early July when the inpoundment became weakly stcatifled. During this time, a stable thermocline persisted for only about 2 weeks early in July at a depth of 6.4 meters. The lack of persistent thermal stability is probably due to: (1) the large volume of cool water in comparison to the size of the surface area available for heating and; (2) the constant withdrawal of hypolinnetic water.

Plots equal dissolved-oxygen concentrations for the deep-water station in Francis F. Walter Reservoir are shown In figure 9. Dissolved-oxygen concentrations were found to be within state standards until late in June, when concentrations in the deeper sections dropped below $5.0 \mathrm{mg} / \mathrm{L}$. Oxygen concentration continued t; drop throughout the summer and by September the entire profile was less than $2 \mathrm{mg} / \mathrm{L}$. Anaerobic and near-anaerobic conditions prevalled untll the autumn overturn early in October.

The temporary low, dissolved-oxygen conditions during the summer of 1981 were undoubtably due to the high biochemical oxygen demand imposed upon the system by the large mass of decomposing vegetation made avallable by the inundation of forest lands due to the ralsing of the pool level. Dissolved-oxyigen profiles for shallow parts of the reservoir, Bear Creek and Lehigh River arms are shown in figure 10 and 11. Much of the volume of the more shallow parts of the reservolr reinalned anoxic or nearly so throughout the season of high biological activity. 


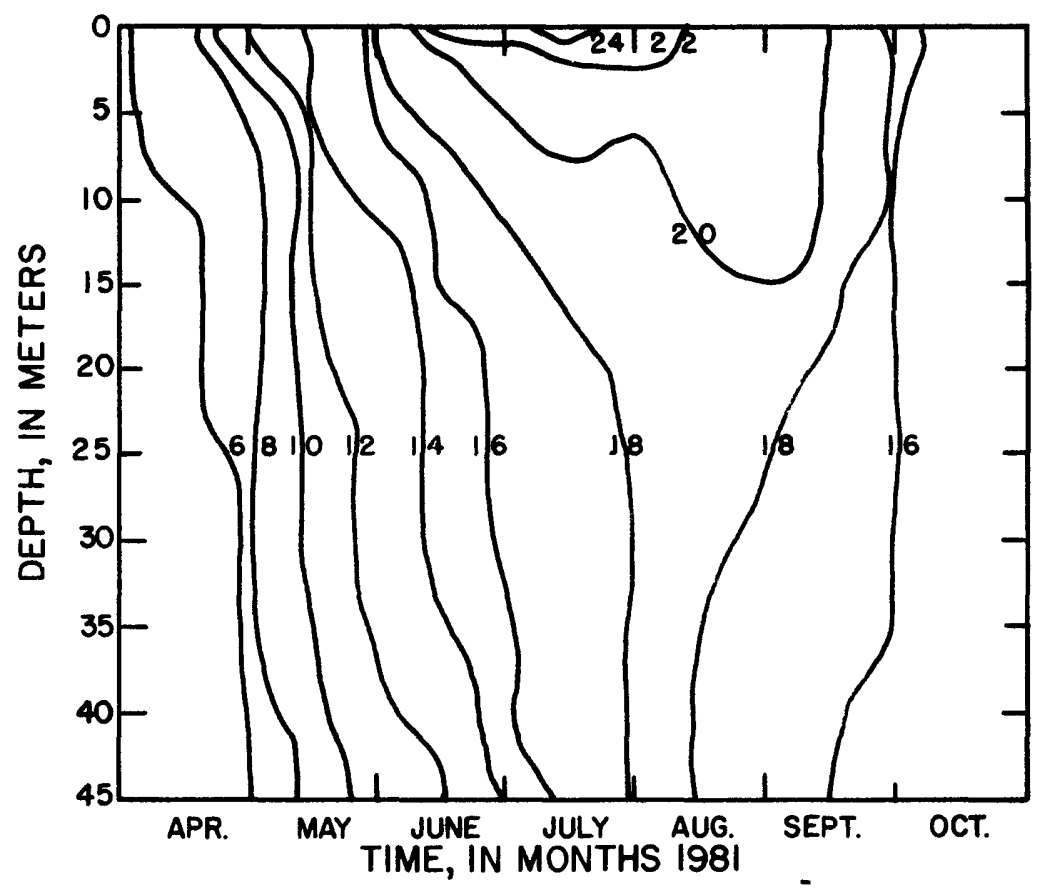

Figure 8.--Plots of equal temperature, spillway pool

(station 4106560754313).

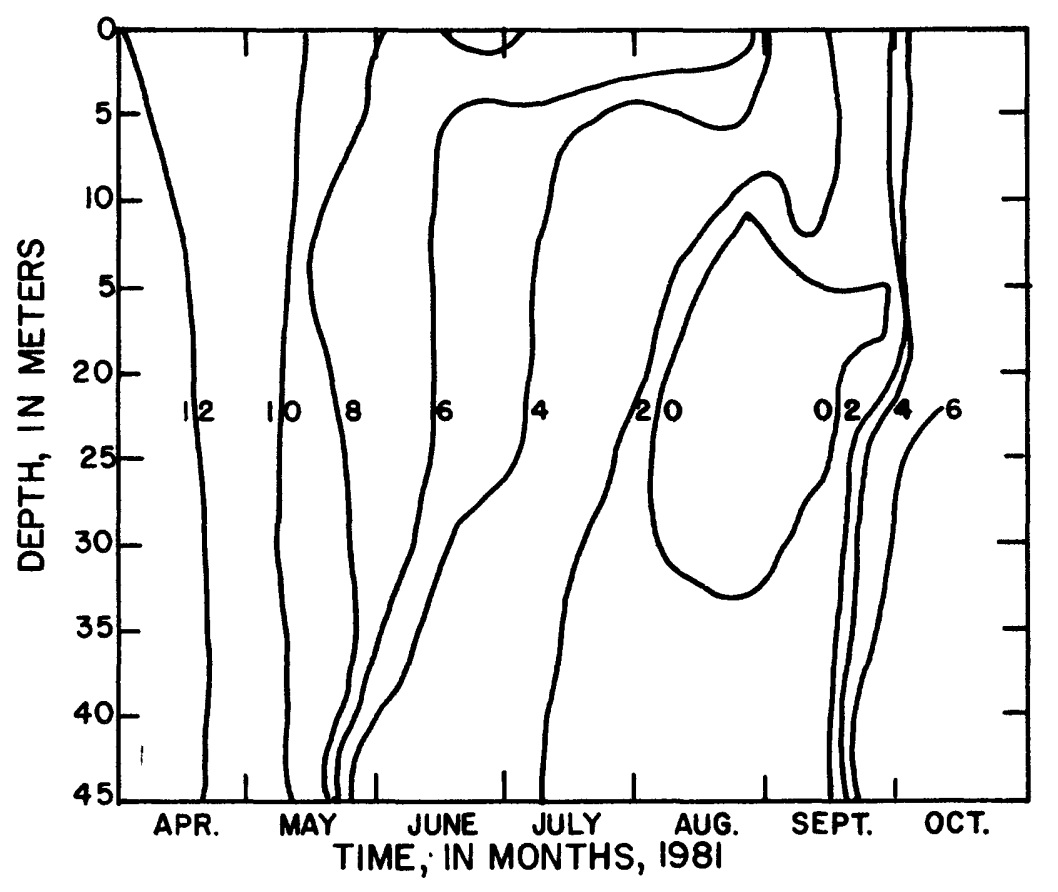

Figure 9.--Plots of equal dissolved oxygen, spillway pool

(station 4106560754313). 


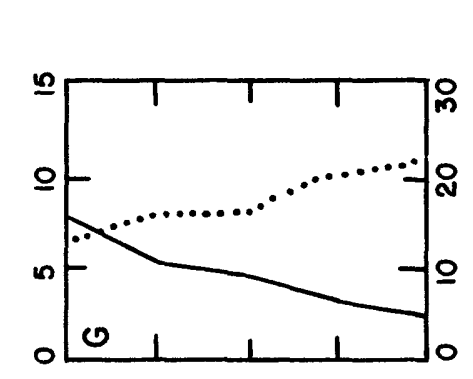

$\infty$

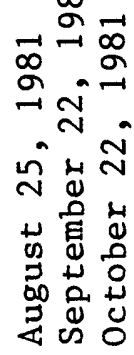

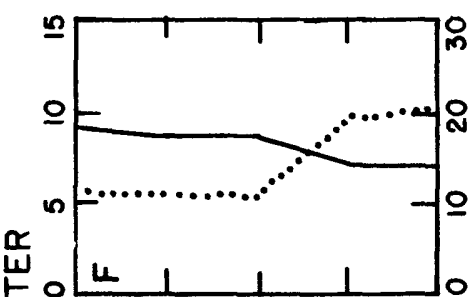

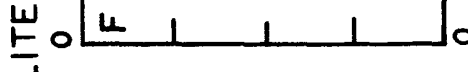
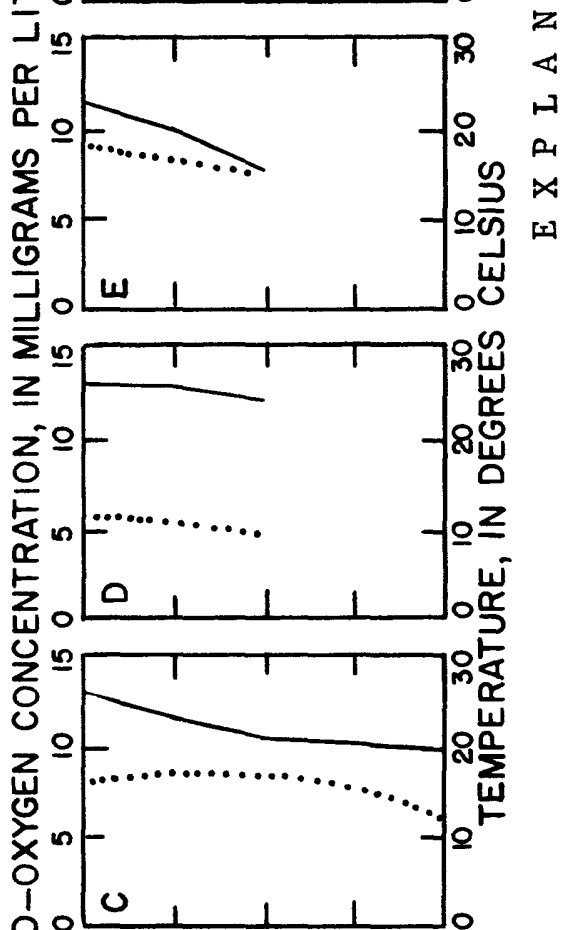

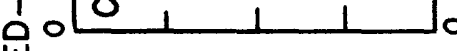
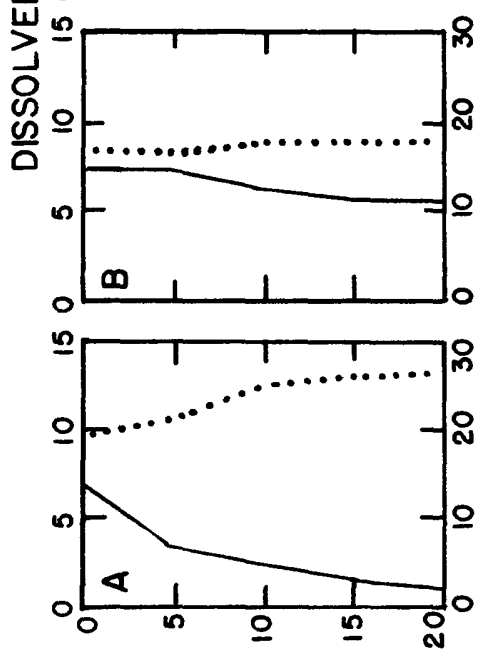

$1 \exists \exists y N N^{\prime} 30 \forall y+4 n S$

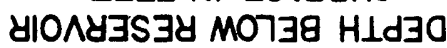

되 饤

$\rightarrow \vec{\infty}$

o

$\rightarrow \infty$

बำ ป

空冧

$\sum \stackrel{5}{5}$

๓ $A$

$\stackrel{5}{0}$

$\infty$

ชิ

ठ

$\stackrel{\pi}{\pi}$

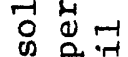

की 윰

苩昰安

$: \mid \varangle$

竞

ร

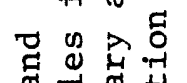

कृ

岳出苛

更

出

i

D政

$\gg$ 㟧

可出 0

की 會

年岪出

응

웅

$\stackrel{0}{3}$

点

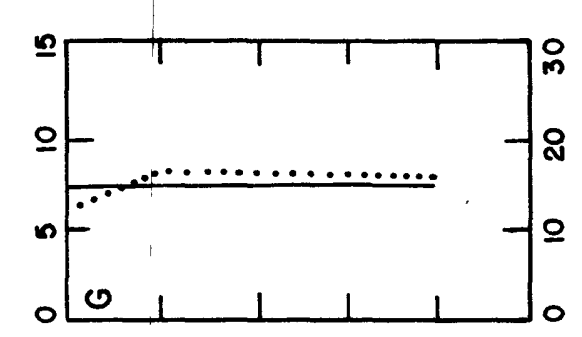

क

- $\stackrel{-\infty}{\infty}$

赵

$\min ^{2}$

กับ

山亘

की

$\infty$

运㻉

되 떤

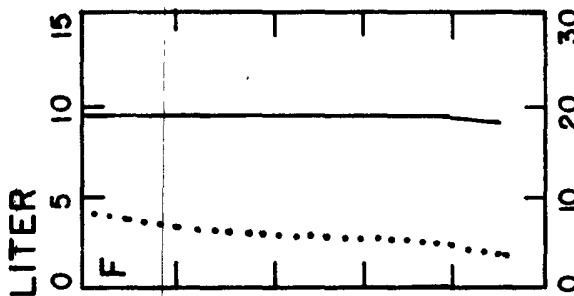

$\rightarrow \vec{\infty} \vec{\infty}$

๙

$\rightarrow$

.

$a \rightarrow-1$

$\rightarrow$ \&

주옳 곡

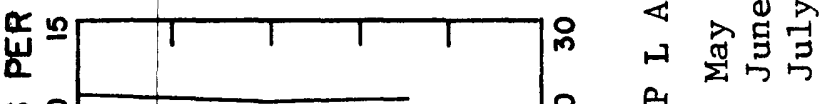

ค U

只

क

त्र

ป

$\rightarrow \pi^{\pi}$ ก

-1

का

क

年

: 14

党

F⿻

- 0 岃

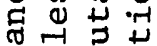

- 1 ह

ट 4 出

$\mathbb{D}_{0}$ 近出

ति के

100

,

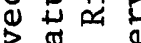

त्र

o

क्ते

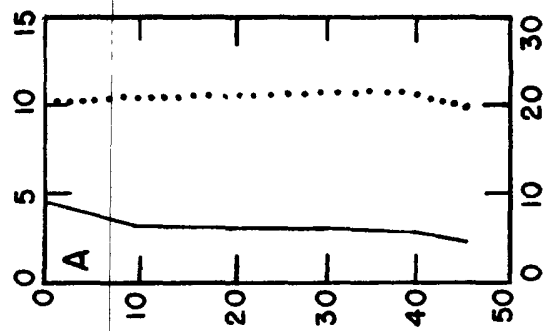

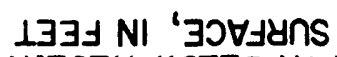

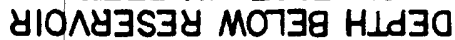




\title{
Transparency
}

Water transparency or visibility was measured with a Secchi disk. The Secchi disk provides a means of comparing the light transmission in similar budies of water or the same body of water at different times. The depth of light penetration is critical to the depth of the euphotic zone and the productivity of the water body.

The maximum, minimum, and mean values of Secchi disk visibility are shown in table 14. Highest nean visibilities were observed in Bear Creek arm and the lowest in the Lehigh River arm. Higher turbidities in the Lehigh River arm appear to be due to the higher density of phytoplankton (fig. 12). The higher phytoplankton densities are probably a product of higher concentrations of avallable phosphorus.

\section{Chemical Characteristics}

\author{
Value of $\mathrm{pH}$
}

The desired $\mathrm{pH}$ range of 6.0 to 3.5 is not being met. The $\mathrm{pH}$ values were generally in the range of 5.0 to 6.0 , with extremes of 4.8 and 6.2 in the main body of the reservolr. The $\mathrm{pH}$ values in the two embayments reflect the water quality of the tributaries. The most acidic water is in the Bear Creek pool while the water in the Lehigh River pool is somewhat less acidic. During August and September when the reservoir was nearly amoxic, there is evidence of $\mathrm{pH}$ depression throughout the reservoir ( $f$ ig. 13). The lower $\mathrm{pH}$ was probably due to the raised pool and subsequent decomposition of the inundated vegetation, resulting in an increase in carbon dioxide $\left(\mathrm{CO}^{2}\right)$, which combines with water $\left(\mathrm{H}_{2} \mathrm{O}\right)$ to form carbonic acid $\left(\mathrm{H}_{2} \mathrm{CO}_{3}\right)$. Measurements of $\mathrm{pH}$ during the spring of 1982 indicate a continued and increasingly more acidic condition.

The vertical distribution of $\mathrm{pH}$ displays a phenomenon referred to as a negative heterograde distribution; that is, $\mathrm{pH}$ decreases with depth and then actually rises before reaching the bottom. This dichotomous curve, first described by Yoshimura (1932) in a number of soft-water lakes in Japan, is belleved the result of a hypolimnetic oxygen deflcit and the diffusion of alkaline earth and ferrous and bicarbonate alkalinity from the sediments.

The actdic condition is a constraint on productivity and diversity of the biota. While the specific effects of low $\mathrm{pH}$ on life in the Francis E. Walter Reservoir are beyond the scope of this study, the generalized effects may be sumarized as follows:

The inhibition of bacteria at low $\mathrm{pH}$ can have profound effects throughout the aquatic ecosystem. Detritus removal, conservation of energy, nutrient recycling, primary production, detritivore production and thus production at higher trophic levels can all be affected by changes in microbial activity. In the pH range of 6.0 to 5.5, there is a reduction in the number of species able to survive and a loss in ability to withstand stress in the surviving species. At a $\mathrm{pH}$ of 5.5 to 5.0, even fewer organisms can survive, but a few pH-tolerant organisms may become abundant. Less than $\mathrm{pH} 5.0$ the decomposition of organic detritus will be severely impaired and most fish species will be eliminated. (Hendry and others, 1979). 


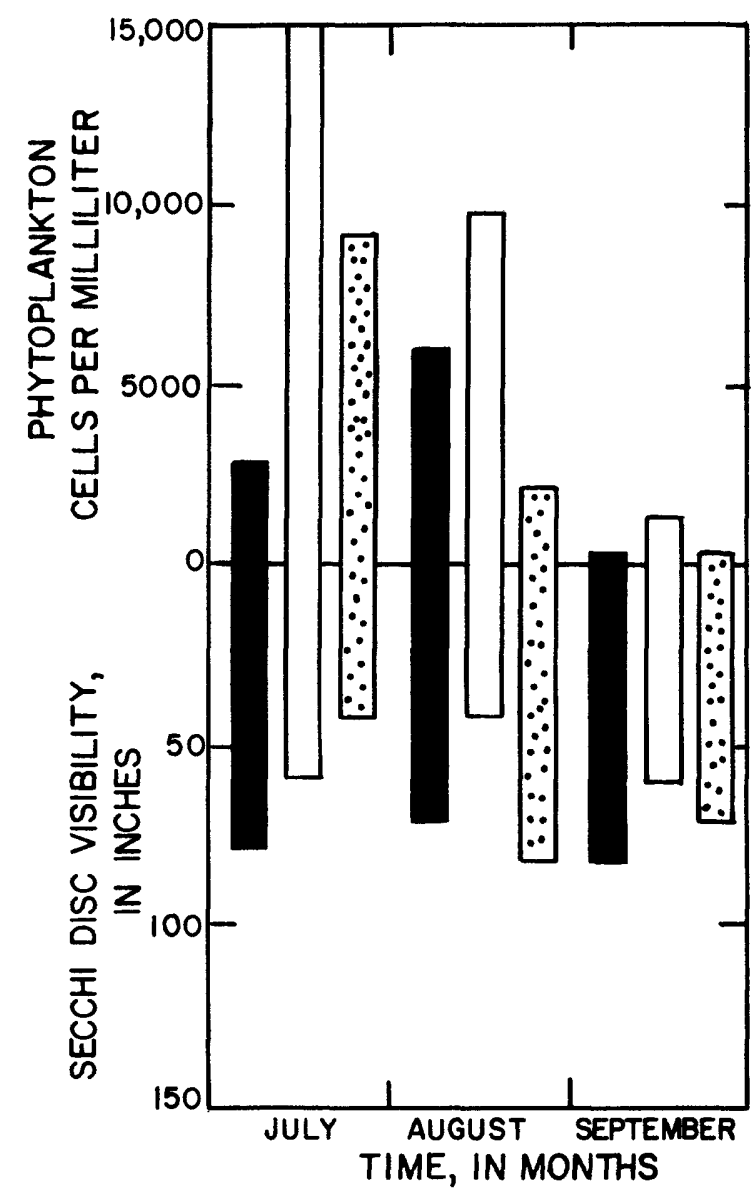

EXPLANATION

Bear Creek

$\square$ Lehigh River

:-::9 Spillway

Figure 12.--Influence of phytoplankton density on Secchi dic visibility.

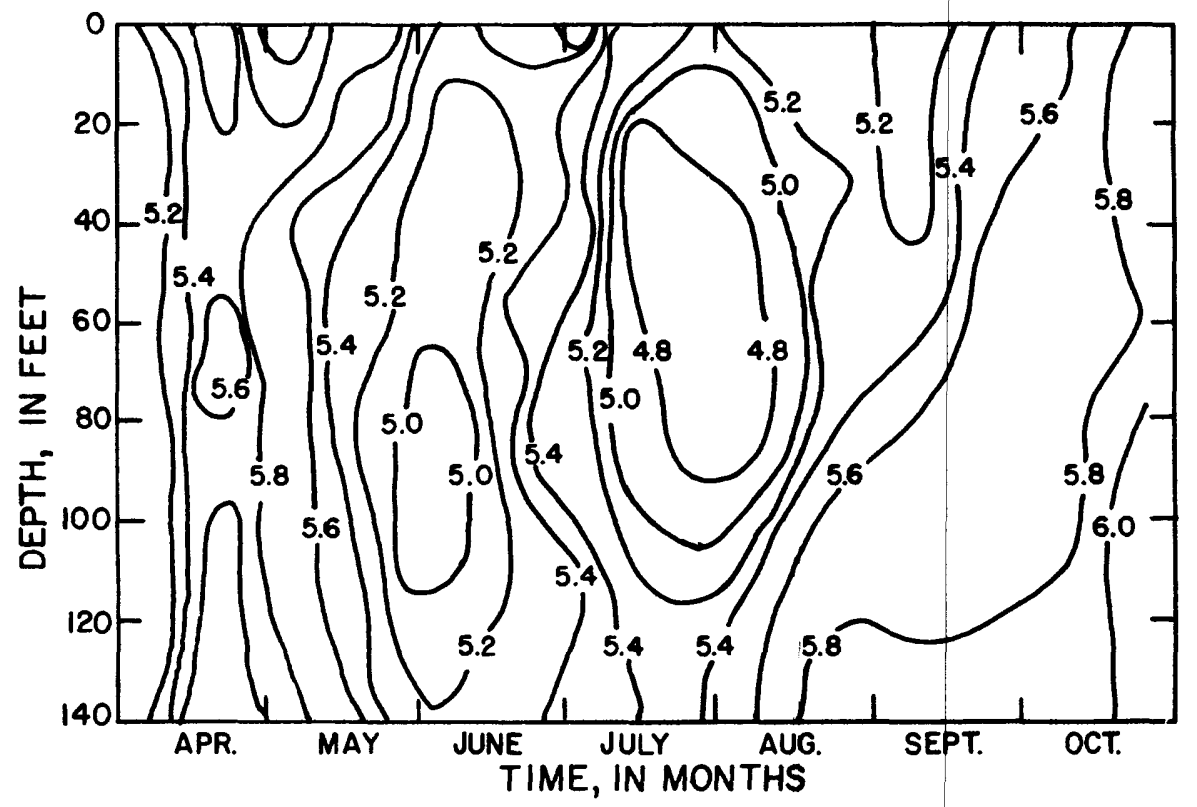

Figure 13.--Depth-time diagram of plots of equal $\mathrm{pH}$ at spillway pool site in 1981. 


\section{Specific Conductance and Dissolved Solids}

Specific conductance or the ionic strength of the water in the reservoir is typical of the waters draining the sandstone soils of the Pocono Plateau. The specific conductance ranged from 45 to 62 micromhos. The higher value was measured in the bottom waters during the late summer.

\section{Nitrogen}

Nitrogen occurs in natural water as dissolved organic nitrogen and as inorganic nitrogen in the form of ammonia, nitrite, nitrate, and elemental nitrogen. Nitrogen is one of the major essential elements for plant growth and is also one of the principal elemental constitutents in amino acids, peptides, proteins, urea, and other organic compounds.

Laboratory tests by Vollenweider (1968), indicate that ammonia and nitrate are the two forms of nitrogen most readily used by planktonic algae. Wang and others (1973) reported that during periods of maximum algal growth, ammonia nitrogen was the preferred source of nitrogen.

The concentration of the various forms of nitrogen in the reservoir is not high and presents no water quality problems. Total nitrogen concentrations in the surface water of the reservoir ranged from $1.5 \mathrm{mg} / \mathrm{L}$ to $5.0 \mathrm{mg} / \mathrm{L}$. It is significant that an average of 80 percent of the total nitrogen is in the organic form. As summarized in table 13, nitrogen avallable for plant uptake or the ammonia and nitrite plus nitrate fraction is very small, averaging 0.06 $\mathrm{mg} / \mathrm{L}$ for both components. Concentrations of nitrogen were highest in the Lehigh river arm.

\section{Phosphorus}

\section{Phosphorus and Algal Growth Potential}

Phosphorus does not occur free in nature but is found in the form of phosphates in several common minerals and is a constituent of all living matter. Like nitrogen and carbon, phosphorus is one of the essential elements for cellular growth. Because phosphorus is an essential element and is generally available in small quantities, it is of ten the limiting factor in the development of summer algae blooms.

The mean total phosphorus content of the surface waters of most Pennsylvania lakes ranges from 0.01 to $0.03 \mathrm{mg} / \mathrm{L}$ (Barker, 1978; Ulanoski and others, 1981). Total phosphorus concentrations of the surface waters of Francis E. Walter Reservoir ranged from $0.002 \mathrm{mg} / \mathrm{L}$ to $0.025 \mathrm{mg} / \mathrm{L}$, with a mean of $0.015 \mathrm{mg} / \mathrm{L}$. Mean concentrations were slightly higher at the Lehigh River pool sampling site. Bottom concentrations were slightly higher than surface waters at all sites, except the Lehigh River pool.

Dissolved orthophosphate $\left(\mathrm{PO}^{-3}\right)$ is the specie of phosphorus most readily used by aquatic plants in photosynthetic processes. Concentrations of dissolved orthophosphorus averaged $0.002 \mathrm{mg} / \mathrm{L}$ at the Spillway pool and Bear Creek pool, and $0.003 \mathrm{mg} / \mathrm{L}$ at the Lehigh River pool. The means and ranges of total, total ortho, and dissolved ortho phosphorus found in this investigation are given in table 14 . 
Algal Growth Potential

Algal growth potential (AGP) is a laboratory test that determines the maximum algal mass (dry weight) of a test algal species (Selenastrum capricormatum) that can be grown in native water in a controlled environuent. While the test does not necessarily reflect natural conditions, it does provide a means of comparing different waters or the growth potential of the same water on different dates.

Algal growth potential was determined for the surface water at the three reservolr sites from June through October 1981. The results, summarized in table 13, indicate that AGP ranged from 0.2 to $0.7 \mathrm{mg} / \mathrm{L}$, and was similar for all sites, and had no discernable seasonal trends. According to a classification study by the Environmental Protection Agency (Miller and others, 1974) on 49 lakes throughout the United States, Francis E. Walter Reservoir has the potential to be a moderately productive lake solely on the basis of a mean AGP of 0.3 $\mathrm{mg} / \mathrm{L}$ for the July sampling.

The ratio of total nitrogen to total phosphorus ( $N: P$ ) provides evidence of the limiting major nutrient. $N$ : $P$ ratios greater than 10 are generally considered to be phosphorus limited. Based upon the N:P ratios of 18 to 400 measured at Francis E. Walter Reservoir, the system is decidedly phosphorus limited.

\section{Biological Characteristics}

Phytoplankton

The composition and density of phytoplankton communities are a reflection of the trophic state. oligotrophic or nutrient-poor waters are characterized by a relatively low phytoplankton density with infrequent pulses or blooms. Eutrophic or nutrient-rich waters are at the opposite end of the spectrum with overall high densities of cells with frequent blooms. Most impoundments, including Francis $\mathrm{E}$. Walter Reservolr, fall somewhere in between and are referred to as mesotrophic.

The phytoplankton communities of Francis E. Walter Reservoir are characterized by overall moderate densities and diversities. All densities were highest tn the early summer and then leveled off for the remainder of the growing season.

During the growing season, 36 genera were recovered from 12 samples. The genera Included 14 green algae, 11 diatoms, 4 yellow-brown algae, 3 blue-green algae, 2 euglenoids, 1 cryptomonad, and 1 dinoflagellate or fire algae. As expected, the highest densities of phytoplankton were found in the Lehigh River arm. This is attributed to the higher concentrations of available nutrients.

Cyanophyta or blue-green algae clearly dominated the population during the warm water growing season. Anacystis $s p$ and Anabeana $s p$ were the codominant genera. Chlorophyta (green algae) and chrysophyta (yellow-brown and diatoms) were also common surface-water types (tables 15-17). The most common green alga was Ankistrodesmus, while the yellow-brown algae were dominated by Mallomanas sp and Dinokryor! is?. 
Table 15.--Summary of phytoplankton composition at spillway pool, Station 410650754313

\begin{tabular}{|c|c|c|c|c|}
\hline & \multicolumn{4}{|c|}{$\begin{array}{l}\text { (Percent of population) } \\
1981\end{array}$} \\
\hline & July & Aug. & Sept. & Oct. \\
\hline \multicolumn{5}{|l|}{ Chlorophyta } \\
\hline Ankistrodesmus & 2 & 10 & 6 & 20 \\
\hline Scenedesmus & -- & 10 & - & 27 \\
\hline Sphairocystis & - & - & - & - \\
\hline Chiamydomonas & 2 & - & - & 10 \\
\hline Schroederia & 1 & 2 & - & - \\
\hline Micnactinium & 13 & - & - & - \\
\hline Gloeoactinium & - & -- & - & - \\
\hline oocystis & - & -- & - & - \\
\hline Dictyosphaerium & -- & -- & -- & - \\
\hline Kirchnerielza & -- & 1 & - & -- \\
\hline Sphaerocystis & - & - & - & 13 \\
\hline Quadrigula & - & -- & - & - \\
\hline Crucigenia & - & 3 & - & - \\
\hline Clostemiopsis & $\ldots$ & -- & - & - \\
\hline Coelastrum & - & 5 & - & - \\
\hline Total & 18 & 34 & 31 & 70 \\
\hline \multicolumn{5}{|l|}{ Chrysophyta } \\
\hline Bacillamiophyceae & - & -- & -- & -- \\
\hline Melosina & - & 2 & - & 20 \\
\hline Asterionella & - & $i$ & - & 10 \\
\hline Epithemia & - & - & -- & - \\
\hline Fragilaria & -- & -- & 6 & - \\
\hline Tabellaria & 4 & - & -- & - \\
\hline Cyclotella & 1 & 1 & - & $\cdots$ \\
\hline Synedra & - & - & -- & - \\
\hline Navicula & - & - & -- & $\cdots$ \\
\hline Gomphonema & -- & -- & -- & -- \\
\hline Nitzchia & -- & 1 & -- & -- \\
\hline Total & 5 & 5 & 6 & 30 \\
\hline
\end{tabular}

Chrysophyceae

Maliomonas

Dinobryon

Jchromonas

Stenocalyx

\begin{tabular}{rrrr}
10 & -- & - & - \\
-- & 1 & - & - \\
-- & -- & -- & - \\
\hline 20 & 1 & - & - \\
\hline
\end{tabular}

Total

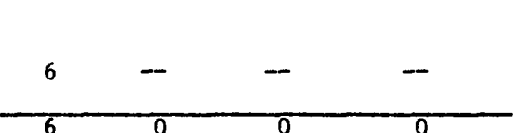

Total

\begin{tabular}{ll}
6 & 0 \\
\hline 6 & 0
\end{tabular}

Euglenophyta

Trache tomonas

Euglena

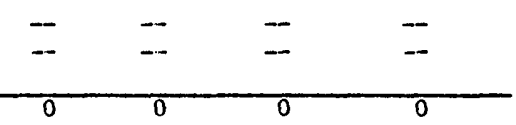

Total

\begin{tabular}{lll}
\hline 0 & 0 & 0
\end{tabular}

Cyanophyta

Anacystis

Gomphosphaeria

Anabrena

\begin{tabular}{cccc}
51 & 46 & -- & - \\
-- & - & - & - \\
-- & 13 & 63 & - \\
\hline 51 & 59 & 63 & 0
\end{tabular}

Total

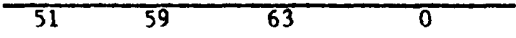

Pyrrhophyta

Peridinium

Total

\begin{tabular}{llll}
- & 3 & - & - \\
\hline 0 & 3 & 0 & 0
\end{tabular}

Total number of

cells, in

$9,100 \quad 2,100 \quad 220 \quad 420$

milligrams per

11ter. 
Table 16.--Summary of phytoplankton compositidn at Bear Creek pool, Station 41108470754306

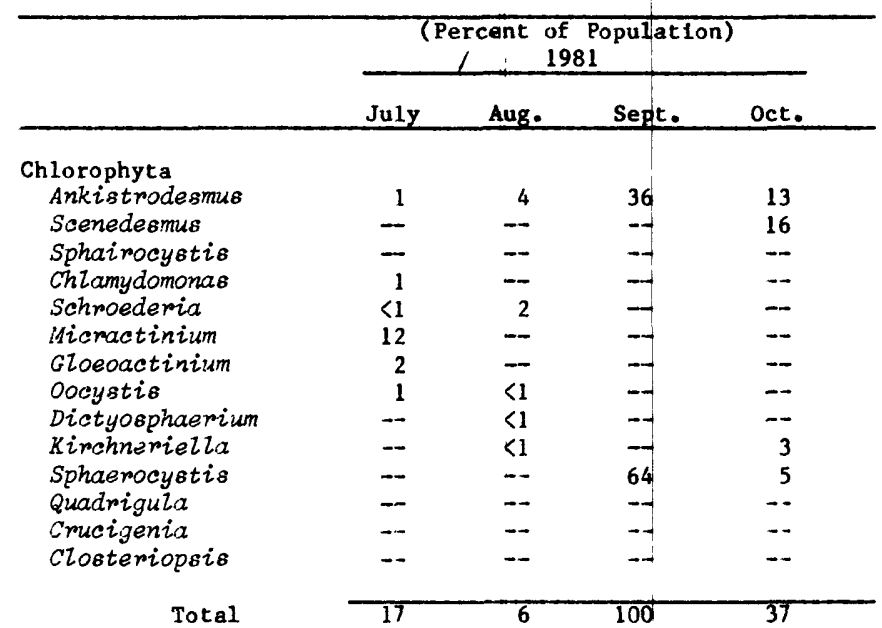

Chrysophyta

Bacillamiophyceae

Melosina

Asterionelza

Epithemia

Eragilaria

Tabelzaria

cyclotella

Synedra

Navicula

Gomphonema

Nitachia

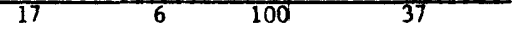

Total

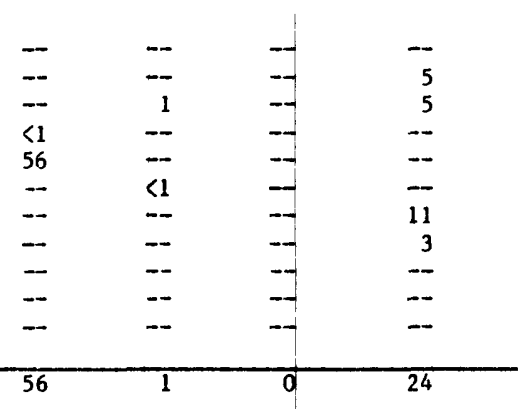

Chrysophyceae

Maliomonas

Dinobryon

ochromonas

Stenocalyx

Total

\begin{tabular}{rrrr}
\hline 56 & 1 & 0 & 24 \\
17 & - & -- & - \\
$<1$ & - & -- & - \\
1 & -- & -- & - \\
-- & $<1$ & -- & - \\
\hline 18 & & 0 & 0
\end{tabular}

Cryptophyta
Chroomonas

Tota 1

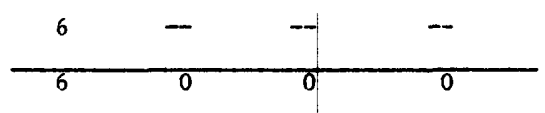

Euglenophyta

Trachelomonas

Euglena

Total

Cyanophyta

Anacystis

Gomphosphaeria

Anabaena

Total

Pyrrhophyta

Peridinum

Total

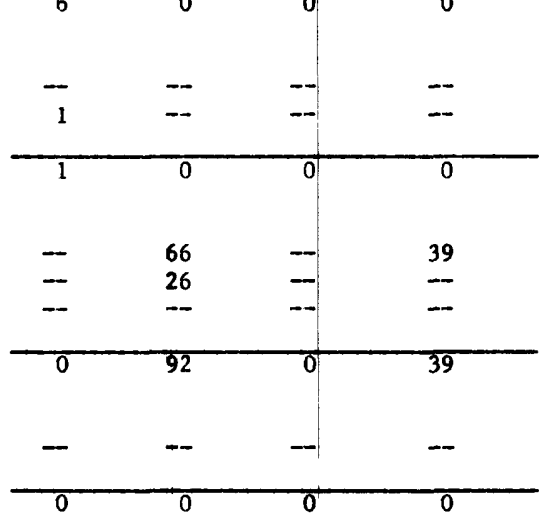

Total number of

cells, In

milligrams per

iiter. 
Table 17.-Summary of phytoplankton composition at Lehigh RIver pool, Station 410750753947

\begin{tabular}{|c|c|c|c|c|}
\hline & & cent 0 & opulat 1 & \\
\hline & July & Aug. & Sept. & Oct. \\
\hline $\begin{array}{l}\text { Chlorophyta } \\
\text { Ankistrodesmus } \\
\text { Scenedesmus } \\
\text { Sphairocystis } \\
\text { Chlamydomonas } \\
\text { Schroederia } \\
\text { Micractinium } \\
\text { Gloeoactinium } \\
\text { Oocystis } \\
\text { Dictyosphasrium } \\
\text { Kirchnemiella } \\
\text { Sphaerocystis } \\
\text { Quadrigula } \\
\text { Crucigenia } \\
\text { Clostsmiopsis }\end{array}$ & $\begin{array}{r}3 \\
2 \\
-1 \\
1 \\
-- \\
- \\
- \\
5 \\
-- \\
-- \\
-- \\
--\end{array}$ & $\begin{array}{l}<1 \\
1 \\
-\overline{<1} \\
<1 \\
-- \\
-- \\
1 \\
-1 \\
-3 \\
-- \\
--\end{array}$ & $\begin{array}{r}2 \\
-- \\
-- \\
-1 \\
-- \\
-- \\
\overline{91} \\
- \\
-- \\
-\end{array}$ & $\begin{array}{l}3 \\
37 \\
- \\
- \\
- \\
- \\
- \\
-- \\
- \\
- \\
- \\
-- \\
-\end{array}$ \\
\hline
\end{tabular}

Total

Chrysophyta

Bacillariophyceae

Melosira

Astemionella

Epithemia

Eragilaria

Tabellaria

Cyclotella

Synedra

Navicula

Gomphonema

Nitschia

Total

\begin{tabular}{llll}
- & -- & - & - \\
$-\overline{2}$ & $<1$ & - & 5 \\
-1 & - & - \\
-- & -- & -- & -- \\
-- & $<1$ & -- & - \\
-- & - & 1 & 5 \\
-1 & -- & - & 5 \\
- & $<1$ & -- & -- \\
-- & -- & - & - \\
\hline 3 & -- & - & 3
\end{tabular}

Chrysophyceae

Mallomonas

Dinobryon

ochromonas

Stenocalyx

$\begin{array}{llll}84 & - & - & - \\ - & 55 & - & - \\ - & - & - & -\end{array}$

Total

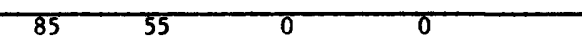

Cryptophyt

Chroomonas

Total

\begin{tabular}{llll}
- & - & - & - \\
\hline 0 & 0 & 0 & 0
\end{tabular}

Euglenophyta

Trachelomonas

Euglena

Total

\begin{tabular}{cccc}
1 & - & 1 & -- \\
- & -- & -- & 3 \\
\hline 1 & 0
\end{tabular}

Cyanophyta

Anacystis

Gomphosphaeria

Anabaena

\begin{tabular}{rrrr}
- & 29 & 2 & 8 \\
- & -- & -- & - \\
- & 7 & - & -- \\
\hline 0 & 36 & 2
\end{tabular}

Total

Pyrrhophyta

Peridinum

Total

\begin{tabular}{cccc}
- & - & - & - \\
\hline 0 & 0 & 0 & 0
\end{tabular}

Total number of

cells, in

milligrams per

1iter. 


\title{
Chlorophy11 a and b
}

Chlorophy11s are complex organic chemicals that utilize light energy from the sun and transform it into chemical energy during the photosynthetic process. Because all living green plants contain chlorophyll, and chlorophyll production depends on light, temperature, nutrients, and other factors, the concentration of chlorophyll is a measure of the production rate.

Concentrations of chlorophy11 a ranged from 0.74 to $15.3 \mathrm{mg} / \mathrm{L}$. Data in table 13 indicate a higher rate of algae production in the Lehigh River pool. This is to be expected because all other indicators show the same trend. As would be anticipated, the lowest concentrations of chlorophyll were found in the Spillway pool because of the depletion of phosphorus, an essential nutrient.

\section{Sanitary Bacteria}

The contamination of recreational waters with fecal material from the gut of warm-blooded animals is of concern because of the associated bacteria and viruses with pathogenic properties. Because of the difficulty in isolating pathogenic organisms, indicator bacteria are used to suggest the possible presence of disease-causing organisms. The indicator bacteria most commonly used are the fecal coliform and fecal streptococcl, both common inhabitants of the intestinal tract of warm-blooded animals. The rationale is that if densities of indicator bacteria are high, then the probability that pathogens are also present increases.

The waters of Francis E. Walter Reservoir were found suitable for all forms of water-contact recreation. Concentrations of fecal coliform and fecal streptococci bacteria were minimal and well within state water-quality criterla (Pennsylvania Water Quality Standards, 1979) 100 percent of the time. Table 14 includes a summary of the bacteria concentrations at the reservoir sites.

\section{GROUND-WATER HYDROLOGY}

\author{
By Charles R. Wood
}

\section{Geology}

The Francis E. Walter Dam and Reservoir are underlain by the sandstones and shales of the lower part of the Pocono Formation and the upper members of the underlying Catskill Formation. These rocks dip northward $5^{\circ}$ to $10^{\circ}$. The bedrock is partly covered by ground moraine of Woodfordian Age, a thin ( 10 to $50 \mathrm{ft}$ ) layer of till consisting of an unsorted mixture of clay, silt, sand, pebbles, cobbles, and boulders. It has a low permeability. Ice-contact stratified drift of Woodfordian Age consisting of sand, gravel, cobbles, and boulders, is present locally. This material has a high permeability.

\section{Ground Water}

The Catskill is a fair aquifer. Carswell and Lloyd (1979, p.11) noted that, "On the average, one of every four wells located, drilled, and developed for high yield will probably produce about $75 \mathrm{gal} / \mathrm{min}$ or more, with $50 \mathrm{ft}$ of drawdown after 24 hours of pumping." They also note that the dissolved-solids 
concentration in water from the Catskill Formation averages about $100 \mathrm{mg} / \mathrm{L}$ and that, "The water ts soft and acidic, and locally contains excessive concentrations of iron and manganese."

Rajising the conservation pool level from the present 1,300-ft elevation to the designed 1,425-ft level will change hydraulic gradients in the aquifers near the reservoir. This change will be large (125 ft) at the reservolr. However, both the land surface and the water table slope steeply (several hundred feet per mile) towards the reservolr; therefore, the change in head caused by raising the pool will be, at most, a few feet at distances of greater than one mile from the reservolr. There are very few wells (probably less than five) near enough to the reservoir to show any rise in water level. These wells will probably increase in yield slightly.

Sore degradation of water quality in the aquifer may occur as reservoir water enters the aquifers when the stage in the reservoir is being increased. Higher than normal concentrations of iron and manganese may occur withtn a few hundred feet of the reservoir. This should be a transient effect, because, after a new equilibrium is reached, water will agatn flow from the aquifer to the reservoir. This will flush the reservoir water that invaded the aquifer back into the pool. None of the existing wells are close enough to the reservoir to be affected.

ENVIRONMENTAL IMPACT AND MANAGEMENT OF A RAISED POOL

The inundation of approximately 1,000 acres of mixed hardwood forest will have negligible impact upon resident terrestrial wildlife such as whitetail deer, bear, turkey, and small mammals. The forest lands bordering the project area are large enough to accommodate the displaced resident wildiffe populations.

A positive effect of the raised and more stable pool is the increased habitat for waterfowl in the drowned forest. Species such as the wood duck should beneft wost. Species of puddle and diving ducks should also utilize the reservolir as feeding and resting areas.

Additional positive effects will be realized to the lake fishery in the form of increased habitat for spawning and nursery areas. Species such as yellow perch and yellow walleye should benefit nost.

Raising the pool level from 1,300 feet to $1,425 \mathrm{ft}$ above sea level will increase the surface area by about 1,200 acres and increase the capactty from 2,000 acre-ft to 72,000 acre-ft. The increased water area will greatly increase both recreational boating and aesthetic appeal. It is significant that the large increase in volume will greatly increase retention time. At the current pool elevation of $1,300 \mathrm{ft}$ and a mean flow of 1,227 acre-ft per day, the retention time is 1.6 days. At a low flow of 119 acre-ft per day, the retention time is 17 days. With the pool capactty increased to 72,000 acre- $\mathrm{ft}$ the mean retention time will be 58 days and at low flow the time will increase to 605 days. As illustrated in figure 14, the trophic status of Francis $E$. Walter Reservoir should inprove as the phosphorus loading rate decreases from 15.1 grains $/ \mathrm{m}^{2} / \mathrm{yr}$ to $1.6 \mathrm{graus} / \mathrm{m}^{2} / \mathrm{yr}$. 


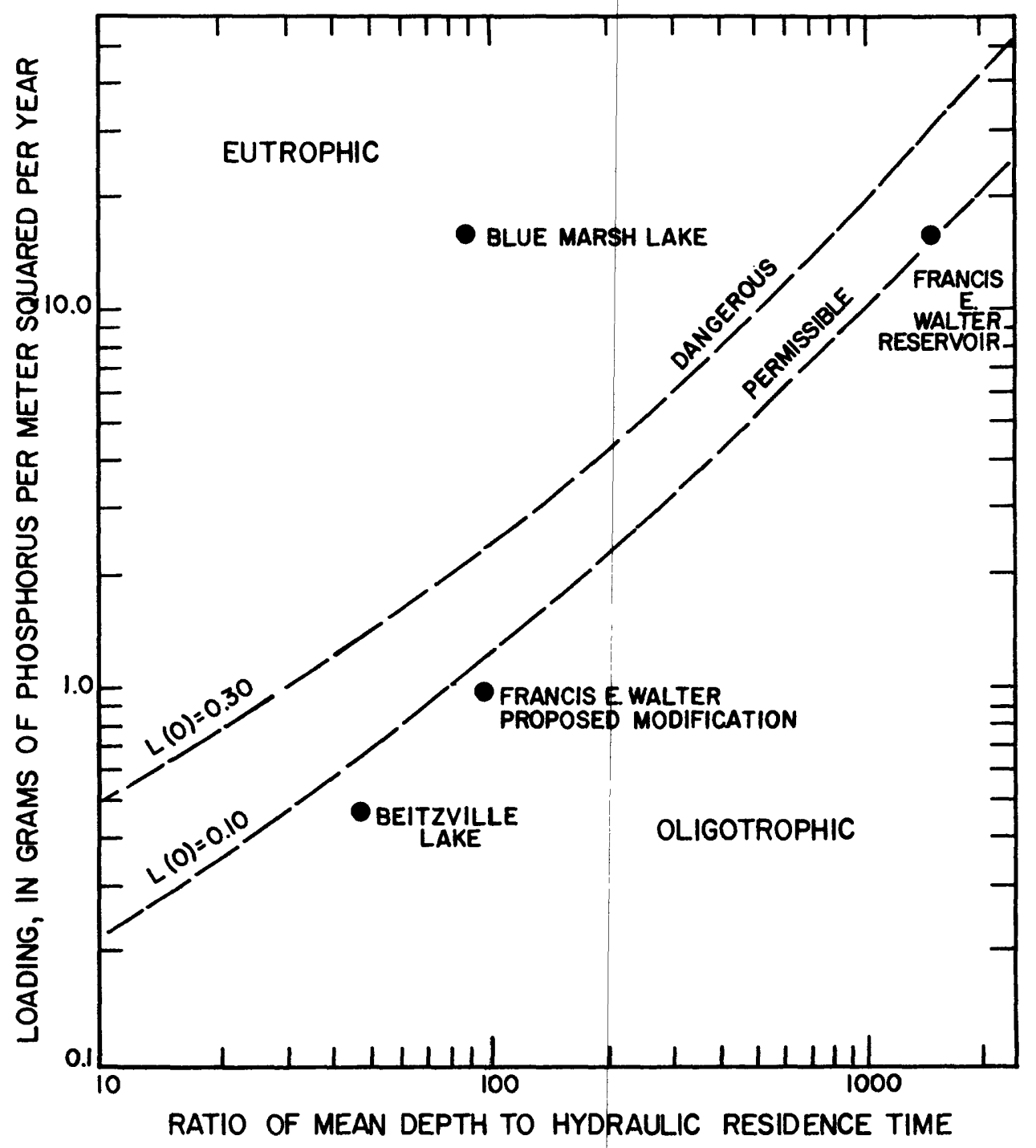

Figure 14.--Total phosphorus loading versus the ratio of mean depth $(\bar{z})$ to hydraulic residence time (TW) and its relation to the eutrophication potential of a lake (from Vollenweider, 1975). 
It is possible that increasing the depth from 45 to $170 \mathrm{ft}$ may, given proper climatic conditions, result in sufficient thermal stability to initiate stratification. An anoxic stratum is also possible until the oxygen demand resulting from the inundated vegetation is satisfied. Coordinated monitoring and use of the selective withdrawal gates would minimize stratification and the volume of anoxic water.

A more stable pool elevation and discharge should also benefit fishery interests downstream of the dam as temperature and flows stabilize. However, withdrawal of anoxic water from the reservoir could have a negative inpact on the quality of water in the tallrace.

Low $\mathrm{pH}$ will continue to be a problem and may tend to restrict the diversity of plants and fish. Increasing the volume and depth will not improve the acldity of the impoundment. Management practices need to be oriented towards maintalning stable pool elevations during the critical spawning and nursery seasons firc the important fishes. Nursery areas will be enhanced if rooted vegetation is allowed to proliferate in the limited shallow areas (less than in ft deep) of the shoreline. One effective way of doing this is to ralse the pool level a few feet during the spawning and nursery season (Apr11-June) and then return the water level to the normal operating elevation for the summer recreation seasion.

\section{SUMMARY}

Past and current water quality data Indicate the waters of the Upper Lehigh River basin are of good quality. The waters are naturally acidic due to a combination of conditions that include acidic precipitation, bogs, and solls low in buffering capacity to neutralize the acidity. The waters are also low in dissolved minerals; and, therefore, are low in overall productivity. Even under these conditions certain species of phytoplankton can and do attain rather high populations.

Reservoir profiles indicate the impoundment stratifies thermally for only a brief period during the summer while dissolved oxygen concentrations were depressed to levels critical to fish throughout much of the reservoir during the warn-water period. The low dissolved oxygen levels are the result of a temporary high biochemical demand brought about by the inundation of forest vegetation.

Tests for enteric bacteria indicate the waters of the impoundment are nearly free of fecal coliform and fecal streptococcus and are therefore sultable for all forms of contact recreation and potable water supplies.

Raising the pool elevation from $1,300 \mathrm{ft}$ to $1,425 \mathrm{ft}$ above sea level is not expected to have any lasting deleterious effects on the water quality. Low $\mathrm{pH}$ will continue to be a problem and restrict the diversity of plants and fishes. Low dissolved oxygen levels found during the summer of 1981 are expected to increase towards saturation as the biochemical demands are satisfied. Increased retention time is not expected to increase future trophic levels because the available phosphorus is presently utilized by the standing crop of primary producers (algae). 
Anderson, J. R., Hardy, Ernest E., Roach, J. T., and Witmer, R. E., 1976, $A$ land use and land cover classification system for use with remote sensor data: J.S. Geological Survey Professional Paper 964, 28 p.

Barker, J. L., 1978, Characteristics of Pennsylvanla recreational lakes: Pennsylvania Department Environmental Resources Bul1. No. 14, 226 p.

Carswe11, I. D., and Lloyd, D. B., Jr., 1979, Geology and groundwater resources of Monroe County, Pennsylvania: Pennsylvania Geological Survey, 4th ser., Water Resource Report 47,61 p.

Geldreich, E. E. and Kenner, B. A., 1969, Concepts of fecal streptococci in stream pollution: Jour. of WPCF, v. 41 , no. 8, pt. 2 , p. 336-352.

Greeson, P. E., Ehlke, T. A., Trwin, F. A., Lium, B. W., and Slack, K. V., 1977, Methods for collection and analysis of aquatic biological and microbiological sanples: J.S. Geological Survey Techniques of Water-Resources Investigations, Book 5, Chapter A4, $332 \mathrm{p}$.

Guy, H. P., 1969, Fluvial sediment concepts: ग.S. Geological Survey Techniques of Water-Resources Investigations, Book 3, Chapter CI, 55 p.

Hendry, S. R., Galloway, J. H., Norton, S. A., and Schofie1d, C. L., 1979, Geological and hydrochemical sensitivity of the Eastern United States to acid precipitation: E.P.A. Report 600/3-80-024, Brookhaven National Laboratury, Upton, N.Y., 100 p.

McCarren, E. F. and Keighton, W. B., 1969. Water quality and discharge of streams in the Lehigh River Basin, Pennsylvania: J.S. Geological Survey Water-Supply Paper 1879-11, 48 p.

Miller, C. R., 1951. Analyses of flow-duration sediment-rating method of computing sediment yield: J.S. Bureau of Reclamation, 55 p.

Miller, W. E., Maloney, T. E., and Greene, ... C., 1974, Algal productivity in 49 lake waters as deternined by algal assays: Water Res. 8: p.667-679.

Pennsylvania Sanitary Water Board, 1979, Chap. 93, Water Quality Standards, Title 25, Part 1, Subpart C, Adopted August 21, 1979, 142 p.

Pollison, D. P. and Craighead, W. M., 1968, Lehigh River biological investigation: Delaware River Basin Commission, Pennsylvania State Department of Health, and Pennsylvanja Fish Commission, 12 p.

Skougstad, M. W., Fishman, M. J., Friedman, I. C., Erdmann, D. E., and Duncan, S. S., 1979, Methods for Determination of Inorganic Substances in Water and Fluvial Sedinents: J.S. Geological Survey, Techniques of Water Resources Investigations, Book 5, Chapter Al, $626 \mathrm{p}$. 
Ulanoski, J. T., Shertzer, R. H., Barker, J. L., and Hartman, R. T., 1981, Trophic classification and characteristics of twenty-six publicly owned Pennsylvania lakes: Pennsylvania Department of Environnental Resources, Pub. No. $61 ., 240$ p.

U.S. Arny Corps of Engineers, 1963, Frances E. Walter Reservoir regulation manual: U.S. Army Corps of Engineers, Philadelphia District, 104 p.

1978, Francis E. Walter Dam and Reservoir Project - Water quality data report (RCS DAEN-CWE-15): J.S. Army Corps of Engineers, Philadelphia District, ibid. 1979, 1980, 1981.

U.S. Department of Commerce, 1976, Climatological data annual summary Pennsylvania: IJ.S. Departinent of Commerce, V. $81,82,83,84,85$, no. 13, $1977,1978,1979$ and 1980.

U.S. Environnental Protection Agency, 1977, Quality criceria for water: U.S. Environinental Protection Agency, 256 p.

Vollenweider, R. A., 1968, Scientific fundamentals of lakes and flowing waters, with particular reference to nitrogen and phosphorus as factors in eutrophication: Organization for Economic Cooperation and Development, Paris DAS/CSI/68.27, 159 p.

1975, Input-output taodels: Schwe1z 7. Hydrol, v. 37, p. 53-84.

Wang, W. C., Sisllivan, W. T., and Evans, R. L., 1973, A technique for evaluating algal growth potential in Illinois surface waters: Illinois State Water Survey Report of Investigation $72,16 \mathrm{p}$.

Yoshinura, S., 1932, On the dichotomous stratiflcation of hydrogen inn concentration of some Japanese lake water: Japanese Journal reological Geogruphic, v. 9, p. 155-185. 